\title{
Development of Individual Geniculocortical Arbors in Cat Striate Cortex and Effects of Binocular Impulse Blockade
}

\author{
Antonella Antonini and Michael P. Stryker \\ W. M. Keck Foundation Center for Integrative Neuroscience, Department of Physiology, University of California, \\ San Francisco, California 94143
}

This study analyzes the morphological changes in geniculocortical axons terminating in the primary visual cortex of the cat, during the period in which, in normal development, the terminals in layer IV undergo an eye-specific segregation. Geniculocortical afferent fibers were filled anterogradely by the Phaseolus lectin (PHA-L) injected into the main laminae of the LGN. After standard immunohistochemical procedures, single axons were serially reconstructed in two or three dimensions. Experiments were performed in normal kittens and in kittens in which retinal activity was continuously blocked by repeated intraocular injections of TTX. In normal kittens, arbors were reconstructed at four different ages $(19,23,30-31$, and 39 days postnatally) spanning the period during which the geniculocortical projection segregates into eye-specific columns in layer IV (LeVay et al., 1978). Results reveal that sparse but widely extending branches characteristic of young arbors are eliminated during normal development at the same time as selected portions of the arbor grow considerably in length and complexity. The terminal arborizations also subdivide into distinct patches of terminals, consistent with the segregation of left and right eye afferents. In TTX-treated animals, axonal arbors reconstructed at postnatal days 23,29 , and 39 show a complexity and extent of terminal arborization similar to that of normal animals, though more variable in size and degree of elaboration. No progressive changes are evident with age. Further, the majority of arbors reconstructed from TTX-treated animals lack the patchy organization typical of normal animals.

In adult mammals with frontal vision, geniculocortical afferents terminate in layer IV of visual cortical area 17 in alternating, eye-specific bands called ocular dominance columns (Hubel and Wiesel, 1972; LeVay and Gilbert, 1976; Shatz ct al., 1977; Anderson et al., 1988). This arrangement of geniculocortical innervation appears to develop from an initial state of overlapping projections by the progressive segregation of the afferents serving the two eyes. The onset and time course of columnar segregation differ among species (Rakic, 1976; Hubel and Wiesel, 1977;

\footnotetext{
Received Sept. 8, 1992; revised Feb. 9, 1993; accepted Mar. 3, 1993.

This work was supported by Grant NIH EY02874 and by NIH Training Grant IT32 EY07120-01. We thank S. Strickland for her invaluable help during the experiments and Dr. J. Horton for his careful critique of the manuscript.

Correspondence should be addressed to Dr. M. P. Stryker, W. M. Keck Foundation Center for Integrative Neuruscience, Department of Physiology, University of California, 513 Parnassus Avenue, San Francisco, CA 94143-0444.

Copyright (C) 1993 Society for Neuroscience $0270-6474 / 93 / 133549-25 \$ 05.00 / 0$
}

LeVay et al., 1978, 1980). Using autoradiographic techniques to visualize the transneuronal transport of tritiated tracers injected into one eye, LeVay et al. (1978) have demonstrated in the cat that the geniculocortical afferents for the two eyes are completely intermixed in layer IV from birth through the first 2 weeks of age. The segregation process begins in the third week and is nearly complete between 8 and 10 weeks postnatally. At this age, the terminal field of the geniculocortical projections in layer IV has acquired an adult configuration charactcrized by periodic, eye-specific clusters.

The cluster-like pattern of geniculocortical innervation in adult cats has been confirmed in studies of serially reconstructed individual axons labeled with HRP from the white matter underlying area 17 (Ferster and LeVay, 1978; Humphrey et al., $1985 \mathrm{a}, \mathrm{b})$. These studies demonstrated that the terminal arborization of mature geniculocortical afferents is subdivided in discrete clusters of collaterals $400-600 \mu \mathrm{m}$ in width, alternating with zones relatively free of innervation. For technical reasons due to the small diameter of axons, intracellular filling cannot be applied to very young animals, and repeated attempts with HRP bulk filling have failed (M. Sur and M. P. Stryker, unpublished observations). Data concerning morphological characteristics of individual geniculocortical afferents prior to and during the course of segregation have been limited (Ghosh and Shatz, 1992).

Two general hypotheses on the reshaping of the developing geniculocortical projections have been suggested (Hubel and Wiesel, 1970, 1977; LeVay et al., 1978, 1980; LeVay and Stryker, 1979; Purves and Lichtman, 1984; Shatz and Luskin, 1986; Friedlander and Martin, 1989). The first hypothesis contends that the terminal arborization of immature geniculocortical afferents passes through a phase of exuberant proliferation characterized by a homogeneous distribution of terminals and a wider terminal ficld compared to mature axons (Hubel and Wiesel, 1970, 1977; LeVay et al., 1978, 1980; LeVay and Stryker, 1979; Shatz and Luskin, 1986). This situation results in a high degree of intermixing of afferents serving both eyes over a common cortical territory. Segregation of eye-specific afferents should then occur through selective pruning of overgrown terminals and/or withdrawal of mispositioned axonal branches. Consistent with this hypothesis is Golgi material in kittens younger than 2 weeks, showing presumed geniculocortical arborizations that span a wide area of layer IV equivalent to two or three eyespecific bands of the adult without gaps (LeVay and Stryker, 1979).

The second hypothesis, based on a study of the morphological characteristics of immature geniculocortical afferents projecting 
to cortical area 18 (Friedlander and Martin, 1989), presumes that the developing terminal arborizations in layer IV are, generally, smaller and less complex than those of the adult and predicts that the maturation process involves a general expansion of the collaterals accompanied by a simultaneous pruning of improper branches. Thus, the segregation of afferents into eye-specific cortical domains should occur by the complete elimination of axonal collaterals impinging on inappropriate cortical domains.

The segregation of ocular dominance columns is thought to involve competition between the two sets of afferents for cortical territory and synaptic space (Hubel and Wiesel, 1970, 1977; LeVay et al., 1978, 1980; LeVay and Stryker, 1979; Purves and Lichtman, 1984; Miller and Stryker, 1990). Neuronal activity in the afferent visual pathway plays an important role in these competitive interactions. Stryker and Harris (1986) have demonstrated that geniculocortical terminals fail to segregate when retinal ganglion cell activity is continuously blocked by repetitive intraocular injections of the voltage-dependent $\mathrm{Na}^{+}$channel blocker TTX during the expected period of segregation.

According to the Hebbian model of neuronal plasticity (Hebb, 1949), this result may be considered an extreme example of disruption of a mechanism that requires activity-dependent interactions for the selective stabilization of a neuronal pathway (Stent, 1973; Changeux and Danchin, 1976). This model would predict that the afferents serving one eye are sorted and their synapses with layer IV neurons stabilized by virtue of their common and temporally coherent pattern of activity. The lack of ganglion cell activity, induced by TTX blockade, would affect the retinally driven geniculocortical projections by eliminating the origin of their patterned activity cues (Arnett, 1978; Arnett and Spaker, 1981; Mastronarde, 1983a,b).

However, the lack of segregation of the geniculocortical afferents in TTX-treated animals may be subject to a second interpretation. It is conceivable that the main function of impulse activity is to promote the general development and growth of neuronal pathways. The results obtained by retinal activity blockade may then be interpreted as a mere expression of a cortical neuronal organization frozen in an immature state (Edwards and Grafstein, 1984; Riccio and Matthews, 1985a-c; Kalil et al., 1986).

A third possible interpretation is that inactivity may be a specific stimulus for geniculocortical afferents to sprout profusely (Brown and Ironton, 1977; Frank, 1987; Fields et al., 1990). Under this hypothesis, the failure to segrcgate results not from the loss of information provided by patterns of activity, but from the overall profusion of the axonal arbors. All these hypotheses are consistent with findings from the bulk labeling of the total geniculocortical afferent projection.

Testing these hypotheses requires a comparison of the morphologies of individual developing geniculocortical afferents in normally raised and TTX-treated animals. To clarify this question, we serially reconstructed geniculocortical afferents anterogradely filled by Phaseolus lectin (PHA-L) injected into the LGN. Our study had two main purposes: to examine the changes in morphology of geniculocortical axonal arbors during normal development, and to test the effects of binocular impulse blockade.

\section{Materials and Methods}

Nine normally pigmented kittens were used for the present study. All were born in the University of California at San Francisco cat colony and were kept with their mother throughout the experiment. Five kittens were reared in a normal environment; their ages at death were postnatal day (P) 19, 23, 30, 31, and 39. Four other kittens underwent bilateral retinal blockade by repeated injections of TTX; their ages at death were P23, P29, and two at P39.

Eye injections. Continuous retinal blockade was produced by repeated injection into the vitreous cavity of a $5 \times 10^{-3} \mathrm{M}$ solution of TTX (Calbiochem) prepared as described previously (Stryker and Harris, 1986). During surgery the animal was kept under halothane anesthesia. An incision in the lateral canthus was made to expose the sclera and a 30 gauge sterile needle was used to puncture the eye over the pars plana If no bleeding occurred, a Hamilton syringe with a 30 gauge needle was then inserted $3-4 \mathrm{~mm}$ deep through the puncture hole and TTX was injected. After the injection, the needle was left in place for $30 \mathrm{sec}$ and withdrawn, and a Q-tip was pressed on the hole for a few seconds to prevent escape of the fluid. The incision was closed with wound clips or suture stitches.

The volume of the TTX solution injected into each eye varied with age starting from $0.5 \mu$ and was increased by $5-10 \%$ for each successive injection. The blockade was monitored by observing a tonic dilation of the pupils and loss of the direct and consensual pupillary reflex to light. It has been shown that this dosage and monitoring regime ensures continuous blockade of retinal activity (Stryker and Harris, 1986).

TTX was injected binocularly at least every $3 \mathrm{~d}$; however, if the animal showed a decrease of pupillary dilation or a slight pupillary response to light in one or both eyes, the TTX injection was carried out earlier. In order to decrease the risk of an acute systemic effect of the toxin, we spaced the injections into the two eyes by a few hours. Table 1 gives the ages at which TTX treatment was started and ended for each animal. lo be effective at disrupting the formation of cortical ocular dominance columns, TTX treatment has to be initiated before or at the beginning of the segregation of geniculocortical afferents (Stryker and Harris, 1986). In animals perfused at a young age (P23, MUC 719; P30, MUC 714; see Table 1) TTX treatment was started at P17, the day after the PHA-L injection into the IGN. This time is well within the initial period of geniculocortical afferent segregation. In the animals perfused at P39 (MUC 720 and MUC 721, Table 1), we had to adopt a different strategy for combining PHA-L injections and TTX treatment since we found that the lectin was degraded after 3 weeks in vivo and therefore could not be injected before beginning TTX blockade. This technical problem was overcome by initialing TTX treatment at P13; the treatment was then interrupted at P26 for $4 \mathrm{~d}$ to allow activity of the retinal ganglion cells to recover, making it possible to visually activate cells in the LGN. At P30, PHA-L was injected into electrophysiologically defined sites of the LGN. The next day, TTX treatment was resumed and maintained until perfusion.

Electrophysiological recordings and PHA-L injections. The aim of the electrophysiological recordings in the LGN was to identify suitable sites within laminae $A$ and $A 1$ for PHA-L injections. The animal was initially anesthetized with a mixture of ketamine hydrochloride $(0.2 \mathrm{mg} / \mathrm{kg})$ and acepromazine $(0.02 \mathrm{mg} / \mathrm{kg})$. Anesthesia was maintained throughout the experiment with halothane (1-2\%) in $\mathrm{N}_{2} \mathrm{O}_{2} / \mathrm{O}_{2}(2: 1)$. The heart rate was continuously monitored and the percentage of halothane in the breathing mixture was adjusted as required. Atropine sulfate $(0.08 \mathrm{mg}$, s.c. $)$ and dexamethasone $(0.8 \mathrm{mg}$, s.c.) were also administered. Pupils were dilated with topical atropine sulfate (2\%), and the nictitating membranes were retracted with phenylephrine hydrochloride (10\%). Tungsten microelectrode penetrations were made between the Horsley-Clarke stereotaxic coordinates AP 2.0-AP 5.0 and ML 5.5-ML 8.0. Once a satisfactory recording site was identified in the main LGN laminae, the microelectrode was withdrawn and substituted with a PHA-L-filled glass pipette. The lectin was iontophoretically injected at the stereotaxic coordinates previously identified by the metal recording electrode, with verification of the depth by recording from the lectin electrode.

Glass micropipettes (10-15 $\mu \mathrm{m}$ tip diameter) were filled from the tip using a vacuum line with a $2.5 \%$ solution of PHA-L (Vector) in $0.1 \mathrm{M}$ sodium phosphate-buffered saline ( $\mathrm{pH} 8$ ). The lectin was injected using a high-voltage current source device (Midgard Electronics) with a positive current $(8 \mu \mathrm{A})$ delivered in $7 \mathrm{sec}$ pulses for 4-10 min. Note that the injection current was higher than that suggested by Gerfen and Sawchenko (1984); we found, in our experiments, that in the developing nervous system lower current intensities were ineffective. Even at high injection currents, only half of the two to four injections made in the LGN successfully produced anterograde labeling in the cortex.

Perfusion and tissue processing. Nine to fifteen days after the PHA-L 
Table 1. Kittens raised with a continuous bilateral blockade of retinal electrical activity obtained by repetitive intraocular injections of TTX

\begin{tabular}{|c|c|c|c|c|c|c|}
\hline \multirow[b]{2}{*}{ Cat } & \multicolumn{2}{|c|}{$\begin{array}{l}\text { TTX } \\
\text { treatment }\end{array}$} & \multirow{2}{*}{$\begin{array}{l}\text { Age at } \\
\text { PHA-L } \\
\text { injec- } \\
\text { tion }\end{array}$} & \multirow{2}{*}{$\begin{array}{l}\text { Age at } \\
\text { death }\end{array}$} & \multirow{2}{*}{$\begin{array}{l}\text { Injected } \\
\text { LGN } \\
\text { laminae }\end{array}$} & \multirow[b]{2}{*}{ Axons } \\
\hline & Start & End & & & & \\
\hline \multirow[t]{4}{*}{ MUC 719} & P17 & $\mathbf{P} 23$ & P16 & $\mathrm{P} 23$ & A & TTX15 \\
\hline & & & & & & TTX16 \\
\hline & & & & & & TTX17 \\
\hline & & & & & & TTX 18 \\
\hline \multirow[t]{6}{*}{ MUC 714} & P17 & P29 & P16 & P29 & $\mathrm{A}+\mathrm{A} 1$ & TTX1 \\
\hline & & & & & & TTX2 \\
\hline & & & & & & TTX3 \\
\hline & & & & & & TTX4 \\
\hline & & & & & & TTX5 \\
\hline & & & & & & TTX6 \\
\hline \multirow[t]{5}{*}{ MUC 720} & P13 & P26 & P30 & P39 & A & TTX7 \\
\hline & P31 & P39 & & & & TTX8 \\
\hline & & & & & & TTX9 \\
\hline & & & & & & TTX10 \\
\hline & & & & & & TTX11 \\
\hline \multirow[t]{2}{*}{ MUC 721} & P13 & P26 & P30 & P39 & A & TTX12 \\
\hline & P31 & P39 & & & & TTX13 \\
\hline
\end{tabular}

For each experimental case, the table lists the ages at which the TTX treatment was started and ended, the age at which PHA-L was injected in the LGN, the age at death, the laminar location of the injection sites in the IGN, and the denomination of the serially reconstructed axonal arbors. All axonal reconstructions were obtained with a three-dimensional computer graphic system.

injection, the animal was killed with an overdose of Nembutal $(60 \mathrm{mg} /$ $\mathrm{kg}$ ) and perfused transcardially with, in succession, ice-cold $0.1 \mathrm{M}$ phosphate buffer; $4 \%$ paraformaldehyde, $0.5 \%$ glutaraldehyde in $0.1 \mathrm{~m}$ phosphate buffer; $4 \%$ paraformaldehyde, $3.42 \mathrm{gm} /$ liter L-lysine (Sigma), 0.55 $\mathrm{gm} /$ liter sodium meta-periodate (Sigma) in $0.1 \mathrm{M}$ phosphate buffer. All solutions had a $\mathrm{pH}$ of 7.4. The brain was removed, the pia was stripped, and a block containing the LGN and the entire caudal pole of the hemisphere where the visual cortex is located was prepared. The block was either postfixed overnight or immediately embedded in gelatinalbumin and cut $(80 \mu \mathrm{m})$ at the vibratome in the frontal plane. Sections were collected in ice-cold potassium-buffered saline ( $\mathrm{pH} 7.4$ ) and processed for immunohistochemistry.

Immunohistochemistry and histology. Indirect immunohistochemical techniques were used to localize PHA-L. Sections were incubated overnight at $4^{\circ} \mathrm{C}$ in a blocking solution composed of $2.5 \%$ bovine serum albumin (BSA), $2 \%$ normal rabbit serum (NRS), and $0.7 \%$ Triton X-100 to reduce nonspecific staining. Sections were then transferred into a solution of $2.5 \%$ BSA and $2 \%$ NRS containing the primary antibody (goat anti-PHA-L; Vector) at a dilution of 1:1000 or 1:2000, and kept at $4^{\circ} \mathrm{C}$ for $24-72 \mathrm{hr}$. Following three washes in PBS (10 min each), sections were incubated for $1 \mathrm{hr}$ at room temperature in a solution containing rabbit anti-goat biotinylated secondary antibody (Vector) in $2.5 \% \mathrm{BSA}, 2 \% \mathrm{NRS}$, and $0.3 \%$ Triton X-100. After three washes in PBS (10 min each), the sections were transferred for 45-60 min into an avidin-HRP complex, washed for at least $1 \mathrm{hr}$, and finally reacted with a freshly prepared solution of $0.05 \%$ diaminobenzidine hydrochloride, $0.7 \%$ nitroammonium sulfate, and $0.3 \%$ hydrogen peroxide. The reaction was monitored at the microscope. All sections were mounted on gelatinized slides, dehydrated in graded series of ethyl alcohol, cleared in xylene, and coverslipped. Sections containing the LGN were stained with cresyl violet for localization of the injection sites.

After reconstruction of geniculocortical axonal arbors, sections were rehydrated and counterstained with cresyl violet in order to determine the cortical layers. The boundaries of the layers were identified according to the cytoarchitectonic criteria of Otsuka and Hassler (1962) and Lund et al. (1979). The border between layers III and IV was placed at the base of the characteristic large pyramidal neurons present in layer III,
Table 2. Kittens raised in a normal environment

\begin{tabular}{|c|c|c|c|c|}
\hline Cat & $\begin{array}{l}\text { Age at } \\
\text { PHA-L } \\
\text { injection }\end{array}$ & $\begin{array}{l}\text { Age at } \\
\text { death }\end{array}$ & $\begin{array}{l}\text { Injected } \\
\text { LGN laminae }\end{array}$ & Axons \\
\hline MUC 736 & P11 & P19 & A & $\begin{array}{l}\text { NN15 } \\
\text { NN16 } \\
\text { NN17 } \\
\text { NN19 } \\
\text { NN20 }\end{array}$ \\
\hline MUC 722 & P14 & $\mathrm{P} 23$ & $\mathrm{~A} 1$ & $\begin{array}{l}\text { NN4 } \\
\text { NN5 } \\
\text { NN6 } \\
\text { NN7 } \\
\text { NN8 } \\
\text { NN9 }\end{array}$ \\
\hline MUC 703 & P17 & P31 & $A+A 1$ & $\begin{array}{l}\text { NN2 } \\
\text { NN3 } \\
\text { D } \\
\text { F } \\
\text { NN11 }\end{array}$ \\
\hline MUC 704 & P18 & P30 & $\mathrm{A}+\mathrm{Al}$ & $\begin{array}{l}\text { NN10 } \\
\text { NN1 } 2\end{array}$ \\
\hline MUC 672 & P24 & P39 & A & $\begin{array}{l}\text { NN1 } \\
\text { NN13 } \\
\text { NN14 } \\
\text { I } \\
\text { J } \\
\text { K } \\
\text { L } \\
\text { M }\end{array}$ \\
\hline
\end{tabular}

For each experimental case, the table lists the age at which PHA-L was injected in the LGN, the age at death, the laminar location of the injection sites in the IGN and the denomination of the serially reconstructed axonal arbors. The axons identified with a single alphabetical letter were reconstructed in two dimensions; the NN series of axons was instead obtained by computer-aided three-dimensional serial reconstruction.

and layer $\mathrm{V}$ was easily identified by the presence of large pyramidal neurons. The subdivision of layer IV into IVa and IVb was not obvious in the young kittens used in this study. Even in our older kittens this identification was sometimes uncertain.

Axonal reconstructions. We limited our analysis to axons with wellfilled arbors that could be followed up to their thinnest terminals. The entire sample of reconstructed axons is listed in Tables 1 and 2. Initial axonal reconstructions (axons D and F in case MUC 703 and axons I, $\mathrm{J}, \mathrm{K}, \mathrm{L}, \mathrm{M}$ in case MUC 672) were performed on axons using traditional methods. Axons were traced in pencil through scrial scctions in two dimensions using a drawing tube and a microscope with a $100 \times$ oil objective at a total magnification of $1000 \times$. Later on, we obtained a three-dimensions computer graphic system, the Neurotrace system described below, able to retain the depth information through a z-encoder attached to the focus knob of the microscope (Neurotrace, InterAction; Passera et al., 1988). All subsequent axons from normal kittens (series NN, Table 2) and the entire axonal sample from TTX-treated kittens (series TTX, Table 1) were reconstructed from serial sections at $1000 \times$ using this system. The drawing tube of the microscope viewed the graphic system screen, and axonal branches were drawn on the screen by digitizing the image seen at the microscope with a mouse. The system was calibrated so that it could measure dimensions both in the $x$, $y$-plane and section thickness in the z-plane. After dehydration, the sections showed a substantial shrinkagc (70-75\%) in thickncss. Knowing the ratio between the original thickness of the section $(80 \mu \mathrm{m})$ and the thickness of the section after dehydration, the system could rescale the drawings to the original dimensions along the z-axis. We did not take into account the minor shrinkage in the plane of the section surface, or the shrinkage of the whole brain after fixation. 

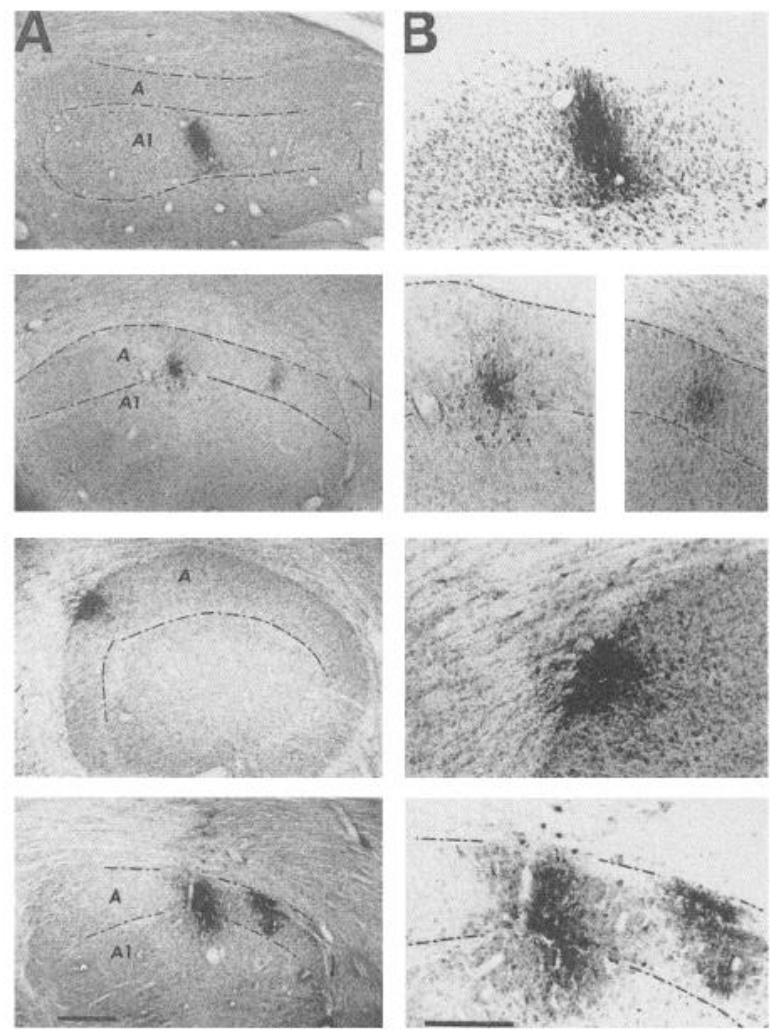

Figure 1. Examples of PHA-L injection sites into the main LGN laminae. The photomicrographs in $A$ show Nissl-stained coronal sections through the LGN in which one or two injection sites are visible. The same sections are presented at greater magnification in $B$ to better show the injection sites. The first three cases are animals raised in normal conditions and are, from top to bottom, MUC 722, MUC 703, and MUC 672 perfused at P23, P31, and P39, respectively. The last case, MUC 714, is a TTX-treated animal perfused at P29 (see Tables 1, 2). $A$ and $A l$ indicate the LGN laminae. Scale bar: $A, 500 \mu \mathrm{m} ; B, 300 \mu \mathrm{m}$.

Axonal reconstructions are shown in two views: in the coronal plane (as reconstructed) and after rotation about the ventrodorsal axis, providing in this case a view tangential to the pial surface. A computeraided drawing program (DIACAD) was used for rescaling all axonal reconstructions to the same dimensions.

For each arbor we made several measurements: (1) the ventrodorsal extension of the arbor along the medial bank of area 17 (VD extension), which expresses the maximal length of the arbor in layer IV and was measured along an axis parallel to the layer; (2) the total linear length of the arborization obtained by the addition of the lengths of all the branches constituting the terminal field of an arbor [only the portion of the arbor located in layers III and IV was considered for this analysisthe axonal trunk, and its bifurcations, was thus clipped just below layer IV; the measurements of the length of the ramifications, as well as number of branch points, average branch length, and coverage area (see below), were taken only for axons reconstructed with the Neurotrace system, which also provides an algorithm for counting the branch points of an axon and for measuring the length of the individual segments in between branch points]; (3) the number of branch points of the terminal arborization in layer IV obtained as described above; (4) the average length of a single axonal branch obtained by dividing the total length of the arborization by the total number of branch points; and (5) the coverage area and density of the terminal arborization in layer IV evaluated from the pial view of the arbor. The terminal arborization was considered to be compressed along an axis perpendicular to the pial surface and to lie in a single plane. The density at each $5 \times 5 \mu \mathrm{m}$ square within the territory covered by the arbor was calculated by summing the total lengths of the portions of all branches that lay within the area enclosed by a circle of $100 \mu \mathrm{m}$ diameter. The coverage area of the arbor was calculated as the area over which arbor density was higher than 2 $\mu \mathrm{m} / 1000 \mu \mathrm{m}^{2}$. This is essentially equal to the area that was within 50 $\mu \mathrm{m}$ from any branch of the arbor.

\section{Results}

PHA-L injection sites in normal and TTX-treated animals

PHA-L injections were made into laminae A and Al of the LGN. Included in the present study are only those experiments in which the injection sites were limited to the main laminae of the LGN and did not invade the medial interlaminar nucleus, the $\mathrm{C}$ laminae, or the monocular segment of layer $\mathrm{A}$. Tables 1 and 2 report for each experiment the age at which PHA-L was injected and the location of the injection sites in intact and TTXtreated kittens, respectively. All injection sites but one involved lamina A, the exception being case MUC 722 in which the lectin was confined to lamina A1. In cases MUC 703, 704, and 714, in which both laminae $\mathrm{A}$ and $\mathrm{A} 1$ were involved in each injection, the major labeling was always in layer $\mathrm{A}$; the few neurons stained in layer Al had probably captured the lectin through their dendrites extending into lamina A (Friedlander et al., 1981).

Figure 1 shows examples of injections in normal and TTXtreated kittens. We could not assess the number of neurons labeled at each injection site because at the center of the injection the labeling was too intense to allow discrimination of the neuronal profiles. It appeared, as reported by Gerfen and Sawchenko (1984), that only neurons heavily labeled at the center of the injection site had effectively incorporated and transported the lectin. We found no cortical labeling in two cases in which neurons at the injection site were faintly labeled.

The cortical labeling was always distributed well within the medial bank of the lateral gyrus and, with the exception of NN1 (see below), all reconstructed axons were away from the 17/18 border, that is, the vertical meridian representation (Tusa et al., 1978). Although we do not know the exact topography of the cortical zones containing PHA-L-stained terminals, we estimate that the cortical label was located in regions at least $5-15^{\circ}$ from the vertical meridian.

\section{General features of PHA-L labeling}

The appearance in layer IV of the terminal ramifications of geniculocortical fibers labeled using PHA-L immunoperoxidase procedures is shown in Figures 2 and 3 for intact and TTXtreated kittens, respectively. No differences were apparent between normal and TTX-treated kittens in the quality of the labeling, suggesting that impulse activity did not interfere with the transport of the lectin. The lectin thoroughly filled axonal branches and their terminals, revealing morphological details such as growth cones (Fig. $2 A, B$ ), varicosities and terminal swellings (Figs. $2 C, 3$ ). The latter could be located at the extremity of an axonal branch, often surrounding a neuronal profile as illustrated in Figure $2 C$, or at the ends of short stalks along a main branch (Fig. $3 A-C$ ). The large varicosities and the terminal swellings are presumably boutons en passant and terminal boutons, although this assertion requires ultrastructural confirmation. However, particularly in younger animals, many swellings were barely detectable and many synapses, identified ultrastructurally in previous work, are not associated with swellings (LeVay and Stryker, 1979), making it impossible to quantify the distribution of synaptic contacts in our material.

Fine collateral branches in layer IV were usually well labeled and easily reconstructed at $1000 \times$ magnification; however, the finest branches sometimes appeared as dotted lines and a small number may have been missed in our reconstructions. We be- 

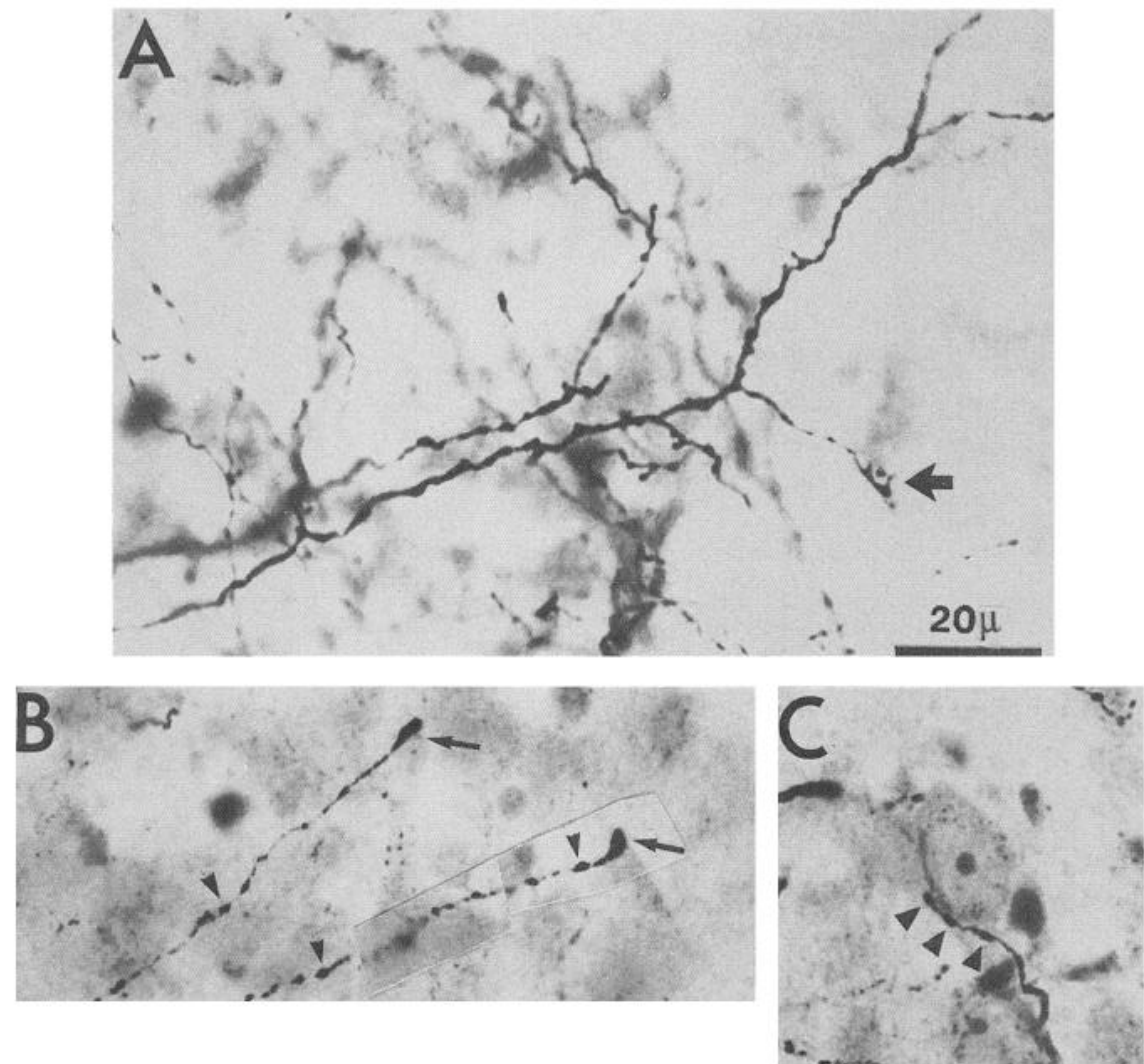

Figure 2. Photomicrographs of PHAL-immunostained terminal arborization of geniculocortical axons anterogradely labeled from the LGN. The case represented is a normal kitten (case MUC 672, P39). The immunostaining procedure is able to reveal in detail the morphological characteristics of the axonal branches such as growth cones (arrows in $A$ and $B$ ) and varicosities (indicated by the arrowheads in $B$ and $C$ ). In $C$, the varicosities appear to follow the outline of a cortical neuron enhanced by Nissl stain. Scale for $B$ and $C$ is the same as in $A$. lieve that this event would not substantially alter the overall morphology of our axonal reconstructions, since we have noticed that when collaterals of comparable size were darkly stained to reveal their full trajectory, they did not form very long projections but extended at most 50-100 $\mu \mathrm{m}$.

The localization and distribution of the terminal fields of geniculocortical axons in layer IV make it likely that most of the arbors reconstructed were of the Y-class, with some of the $\mathrm{X}$-class as well. For the whole sample of axons, the terminal arborization in layer IV was well immunostained and easily reconstructed. In contrast, axonal trunks appeared more faintly labeled in the deepest portion of layer VI and often could not be followed far into the white matter. For this reason we refer to our reconstructions as geniculocortical arbors rather than axons, since it is possible that some parent axonal trunks gave off other collaterals in the deepest portion of the white matter (Ferster and LeVay, 1978; Friedlander and Martin, 1989).

In the next two sections, a description will be presented of the morphological characteristics of geniculocortical arbors reconstructed from area 17 of normal and TTX-treated kittens at
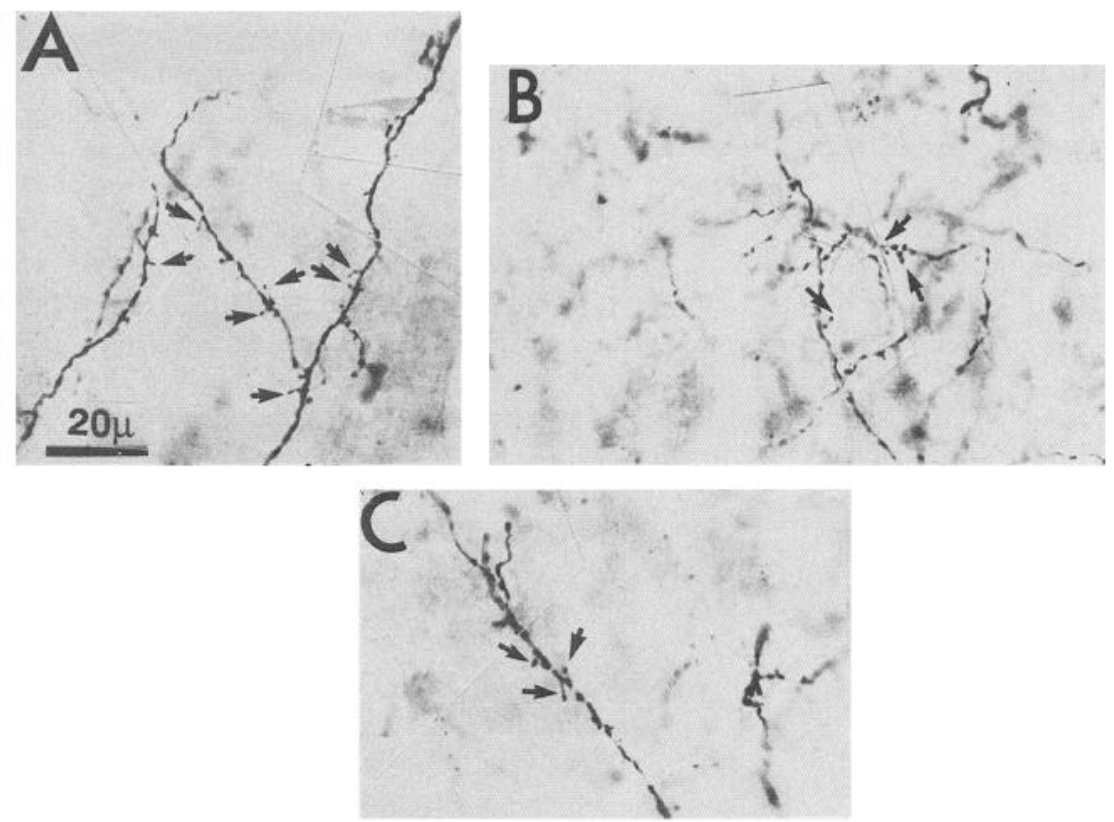

Figure 3. Photomicrographs of PHA$\mathrm{L}$-immunostained terminal branches in layer IV of geniculocortical axons in TTX-treated kittens. The photomicrographs emphasize the resolution afforded by the immunoperoxidase procedure. In $A-C$, the arrows point at varicosities located on short stems arising from main arborizations. 


\section{P19}

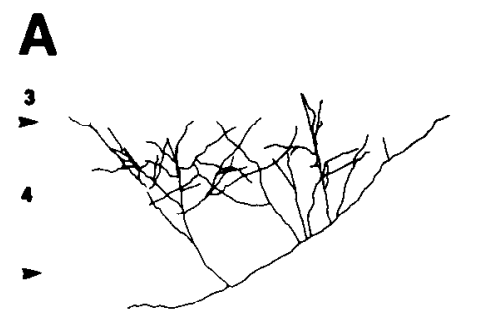

NN15

B

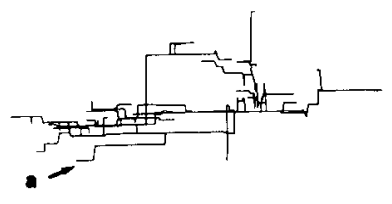

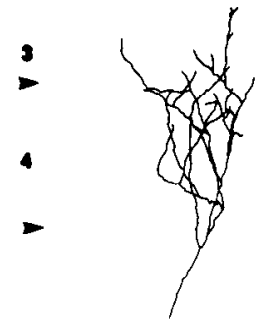

NN16

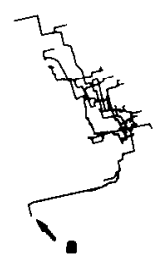

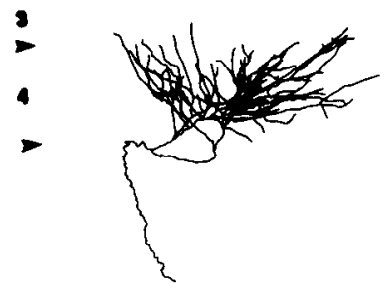

NN17

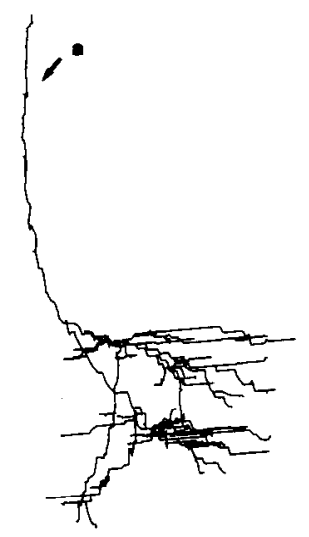

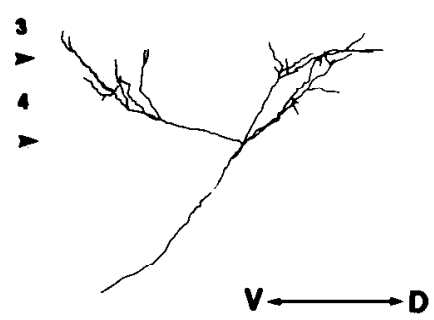

$\mathbf{V}$

NN 19
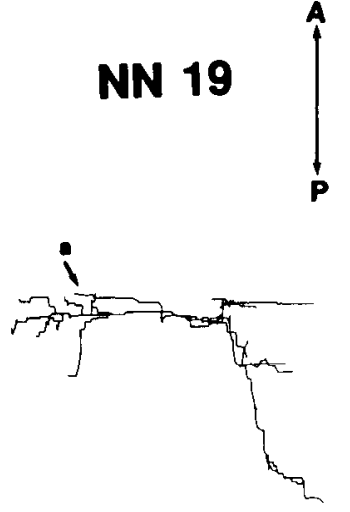

$400 \mu$

Figure 4. Computer reconstruction of PHA-L-immunostained axonal arbors (NN15, NN16, NN17, and NN19) in area 17 of a P19 kitten. Row $A$ shows the arbors as originally reconstructed in coronal view. Row $B$ shows the same axons from a surface view after a $90^{\circ}$ angle rotation along the ventrodorsal axis. In $B, a$ indicates the main axonal trunk as it enters into the gray matter. The scale bar applies to both $A$ and $B$.

four and three different ages, respectively. Subsequently, a quantitative analysis of the arbors, including statistical comparisons within and across the two experimental groups, will be presented.

\section{Development of geniculocortical arbors in normal kittens}

$P 19$. Five arbors were reconstructed from area 17 in a $P 19$ kitten (MUC 736) raised normally (Table 2). Figure 4 illustrates four of the arbors viewed in the coronal plane, in which they were traced (Fig. $4 A$ ) and from the pial surface after a rotation of $90^{\circ}$ along the ventrodorsal axis (Fig. $4 B$ ). With the exception of arbor NN16, which ramified across the entire width of layer IV, the remaining threc arbors appearcd to expand mainly in the superficial portion of the thalamo-recipient layer, suggesting a possible relation with the $\mathrm{Y}$ contingent of the geniculocortical projection (Ferster and LeVay, 1978; Humphrey et al., 1985a,b). In all arbors, the terminals crossed the layer III/IV border and invaded the lower tier of layer III. Note that in arbor NN16 a few branches extended beyond the deep portion of this layer; indeed, at P19 many thin collaterals of axons that were not reconstructed reached layers II and I.

Two general features characterized arbors reconstructed at P19. First, the terminal arborization was broadly extended along the ventrodorsal axis and did not demonstrate any obvious patterned organization, although most of the branches of arbor NN19 were located at its ventral and dorsal extremes. Second, in four of the five arbors (NN15, NN16, NN19, and NN20, not shown) the terminal arborization was constituted by thin col- laterals, very scantly ramified. In this regard, arbor NN17 represented an exception in the group, in that the broad terminal field was strikingly ramified. Another peculiar feature is that the bare axonal trunk of arbor NN17 ran anteroposteriorly for about $800 \mu \mathrm{m}$ across the infragranular layers and a portion of layer IV before it finally ramified and produced the terminal arborization, suggesting that geniculocortical axons may lack precise topographic organization within the white matter.

$P 23$. Six arbors were reconstructed from the medial bank of the lateral gyrus of a P23 kitten raised in a normal visual environment (MUC 722, Table 2). All arbors originated from neurons located in lamina Al of the LGN (see Fig. 1). Figure 5 illustrates four examples of computer reconstructions of the arbors viewed in the coronal plane (Fig. $5 \mathrm{~A}$ ) and after a $90^{\circ}$ rotation along the ventrodorsal axis (Fig. $5 B$ ). In these, and the remaining two axons of the group, the terminal arborization was located in the upper portion of layer IV. In arbors NN4 and NN6 the inital bifurcation of the axon trunk occurred in the lower part of layer IV, but subsequent divisions were identified more superficially in the layer. The terminal arborizations of all axons were mainly constituted of very thin branches, sparingly ramified and yet, with one exception (NN9, not illustrated), were broadly extended along both the ventrodorsal and the anteroposterior axes. As at P19, the collaterals were rather uniformly distributed within the terminal field. Note, however, that collateral branches were preferentially located at the dorsal and ventral extremities of arbor NN5, consistent with the initiation of segregation, although we have no reason to believe 
P23

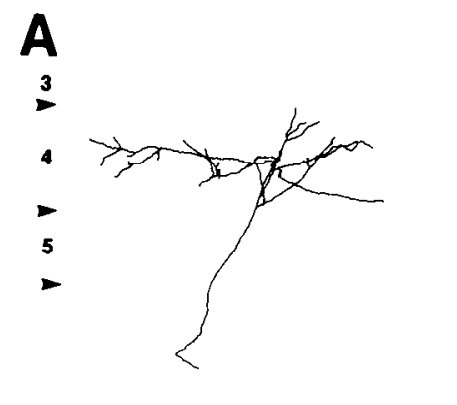

B

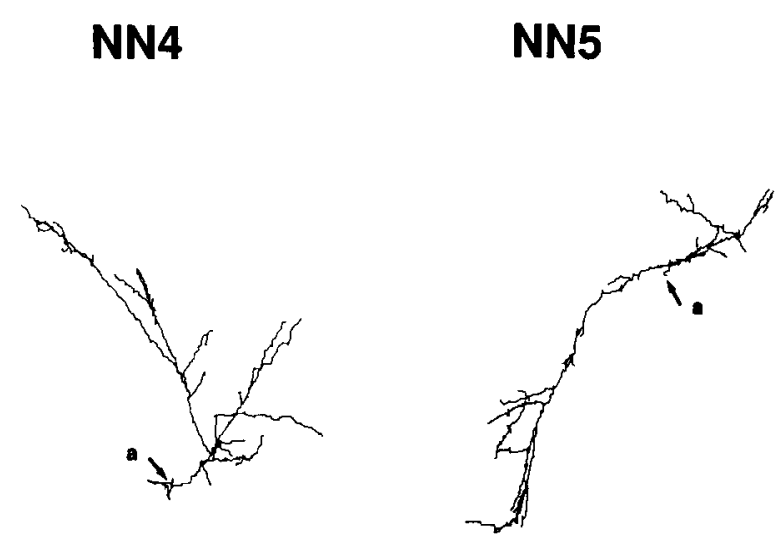

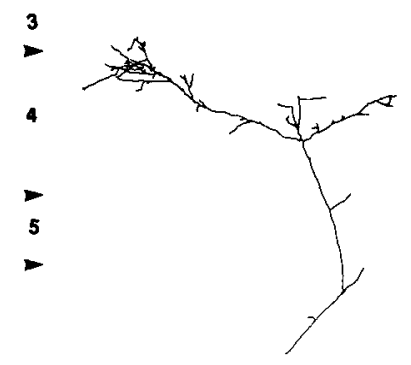
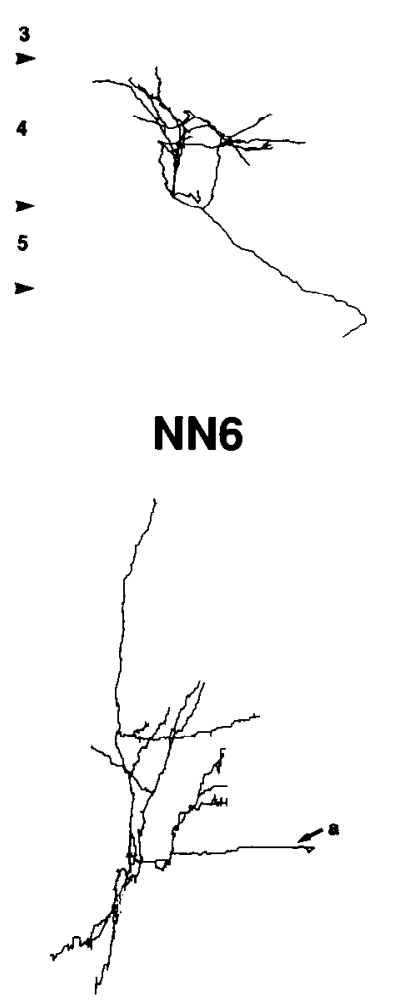
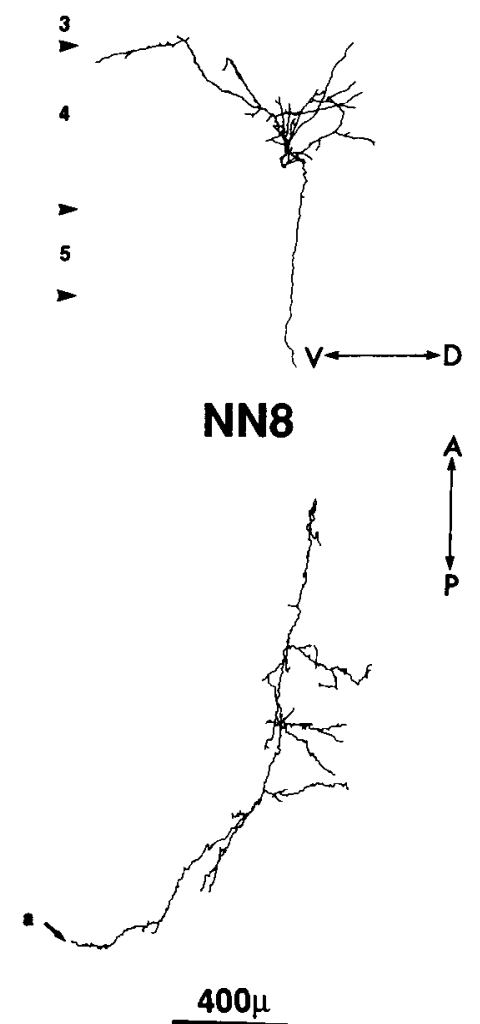

Figure 5. Computer reconstruction of PHA-L-immunostained axonal arbors (NN4, NN5, NN6, and NN8) in area 17 of a P23 kitten. Row A shows the arbors as originally reconstructed in coronal view. Row $B$ shows the same axons from a surface view after a $90^{\circ}$ angle rotation along the dorsoventral axis. All axons are poorly branched and ramify predominantly in the upper portion of layer IV. In $B, a$ indicates the main axonal trunk as it enters into the gray matter. The scale bar applies to both $A$ and $B$.

that such slight concentrations of arbor density (as in NN19 above) are not random.

$P 30 / 31$. Seven well-stained arbors, located in the medial aspect of the lateral gyrus, were selected for serial reconstruction in two normal kittens aged P30 and P31 (MUC 703 and MUC 704 , Table 2). Two arbors were reconstructed in two dimensions (arbors D and F, not shown) and five in three dimensions with the computerized system (NN2, NN3, NN10-NN12; Figs. 6, 7). In MUC 703, two PHA-L injection sites involved both laminae $A$ and $A 1$ of the LGN. Of the three injection sites present in MUC 704, one was confined to layer A and the other two involved both laminae $A$ and A1. Two injection sites from MUC 703 are illustrated in the photomicrograph of Figure 1.

Axons at this age are characterized by great variation in the extension and pattern of terminal arborization. All arbors, except arbor D, ramified mainly within the upper tier of layer IV. In coronal view, two main morphological classes could be identified. One class, comprising arbors NN11 (Fig. 6A) and NN3 (not shown), had a widely extended terminal field $(>1500 \mu \mathrm{m})$, similar to the immature axons previously described. The other class of arbors, comprising arbors D, F, NN2, NN10, and NN12, was characterized by a relatively restricted terminal field (range, $380-630 \mu \mathrm{m})$. This feature represents a major difference from the widely extended arbors found at a younger age.

Another distinctive characteristic of axons reconstructed at $\mathrm{P} 30 / 31$ was the clustered pattern into which the terminal ar- borization was organized. This feature is clearly appreciated when arbors are viewed from the pial surface. From this perspective, the terminal arborization of axons NN10 and NN12 appeared subdivided, along the anteroposterior axis in clusters of terminals. This feature is not present in arbors reconstructed at P23. The two clusters of arbor NN10 originated from a bifurcation of the main axonal trunk; in NN12 the clusters developed instead from a collateral branch originating within layer IV and directed posteriorly relative to the main axonal trunk. The tcrminal field of arbor NN2 was restricted not only along the ventrodorsal but also along the anteroposterior axis, such that the whole arbor was homogeneously distributed over a round-shaped surface. In surface view, arbor NN11 presented two incompletely segregated patches of terminals (indicated by the two arrows in Fig. $6 B$ ). Arbor NN11 was also noteworthy for the presence of a far-reaching, thick and poorly ramified axonal branch running for several hundred micrometers parallel to layer IV. This morphological characteristic was often found in arbors reconstructed at P30/31 and P39 (see arbor NN1, Fig. 10). A photomicrograph of a portion of arbor NN11 (Fig. 7) demonstrates that the long axonal branch that runs ventrodorsally parallel to the III/IV border is almost completely devoid of ramifications.

P39. Eight axonal arbors were serially reconstructed from case MUC 672 perfused at P39. Five arbors were reconstructed in two dimensions (arbors $\mathrm{I}-\mathrm{M}$ ) and three in three dimensions 
P30-31

A

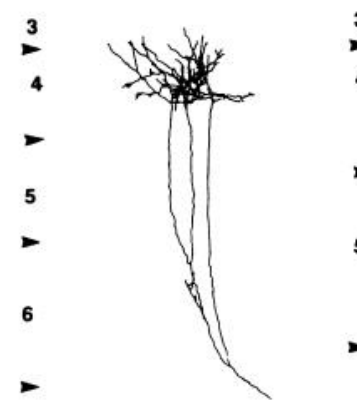

NN10

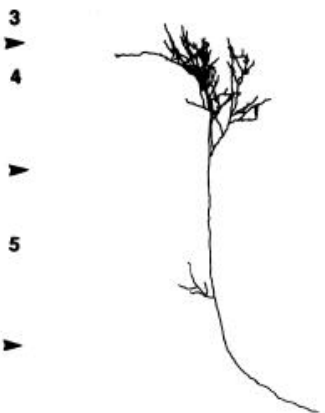

NN12

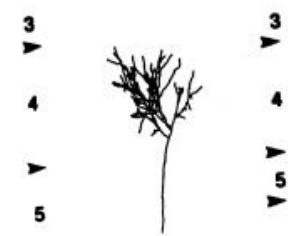

NN2

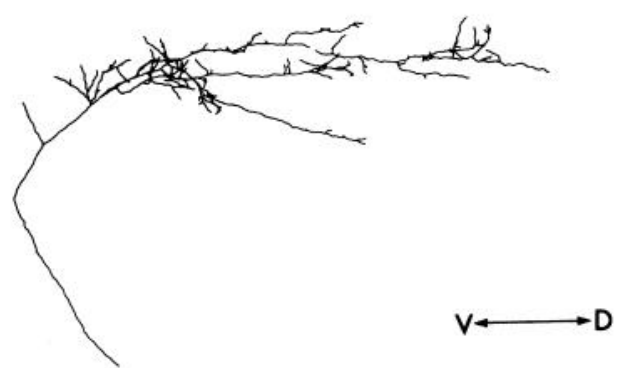

NN11

B
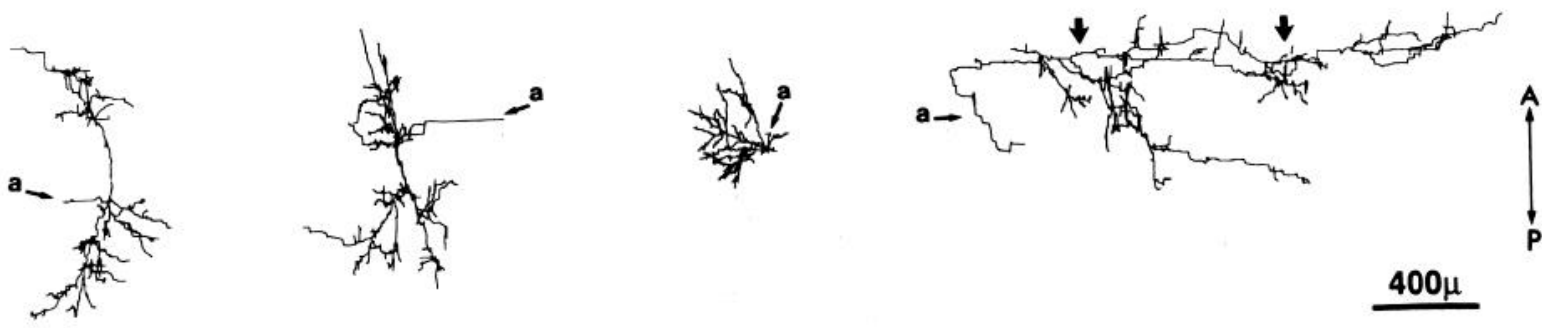

$400 \mu$

Figure 6. Axonal arbors $(N N 10, N N 12, N N 2$, and $N N 11)$ reconstructed from kittens at $\mathrm{P} 30$ and $\mathrm{P} 31$. Axons are shown in coronal view $(A)$ and in surface view $(B)$. Note that the patches of terminals into which the terminal field of axonal arbors $N N 10, N N 11$, and $N N 12$ is subdivided are distinguishable only from the surface view. The patches of terminals in NNIO and NNI2 are separated by a branch totally devoid of collaterals, whereas in arbor $N N I I$ two patches of terminals, indicated by the arrows, are not completely isolated and the cortical territory between them receives a few collateral branches. In $B, a$ indicates the main axonal trunk. The scale bar applies to both $A$ and $B$.

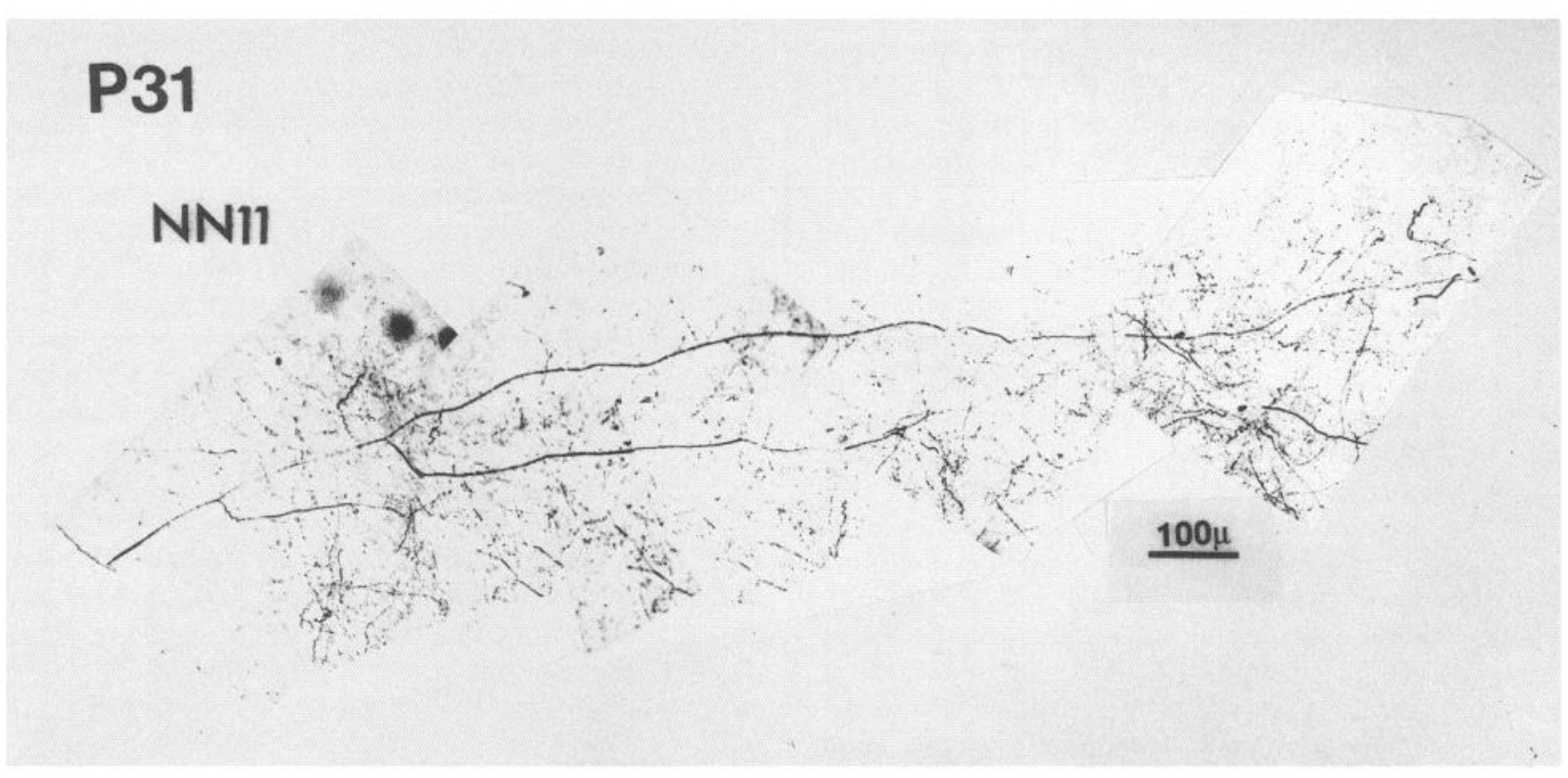

Figure 7. Composed photomicrograph of a portion of arbor NN11 shown in the computer reconstruction in Figure 6. Note the long projecting axonal branches running ventrodorsally and parallel to the cortical layers. 


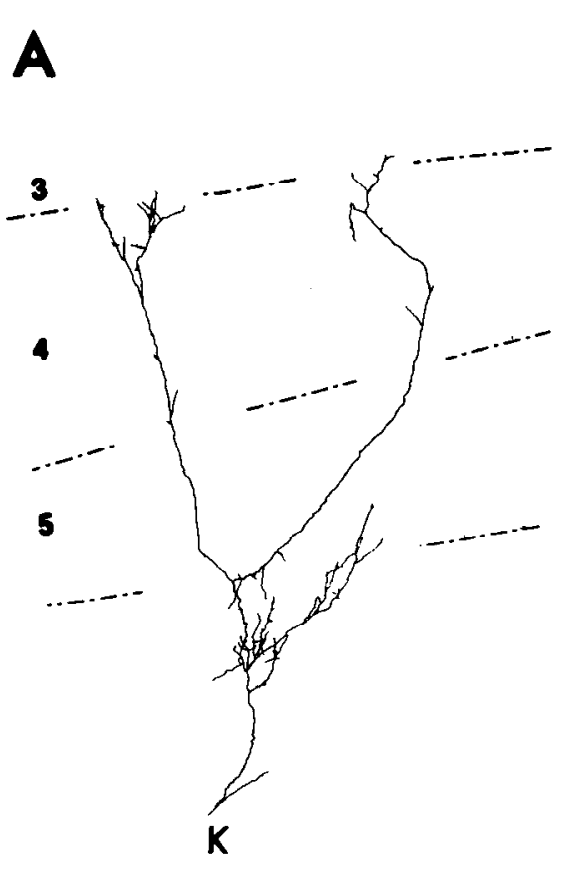

D

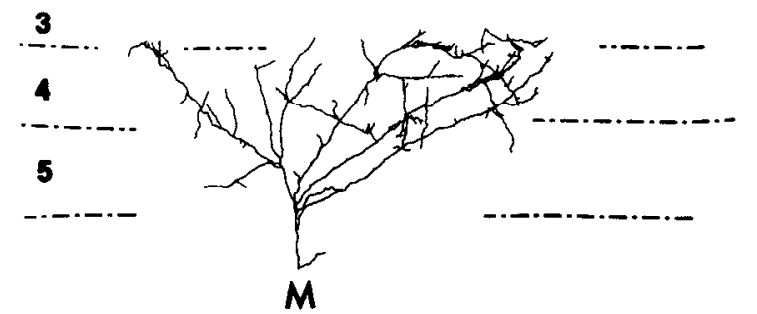

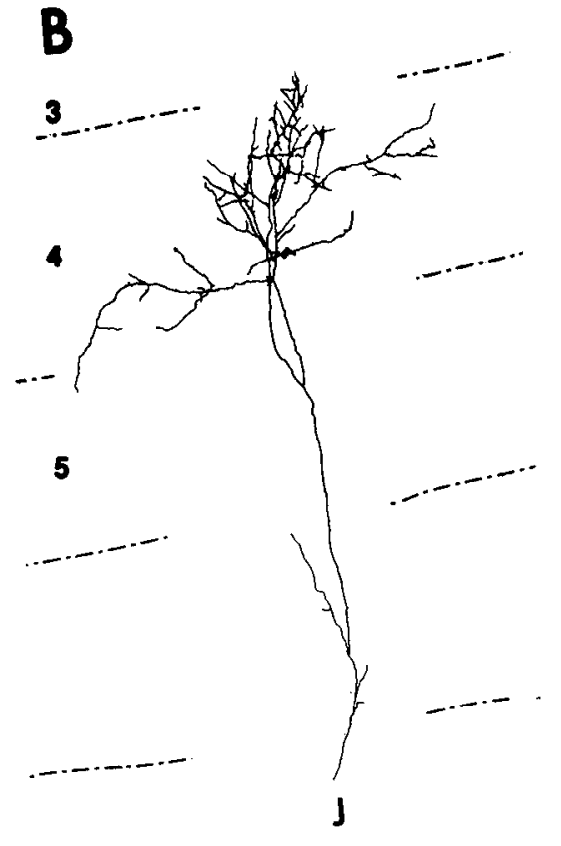
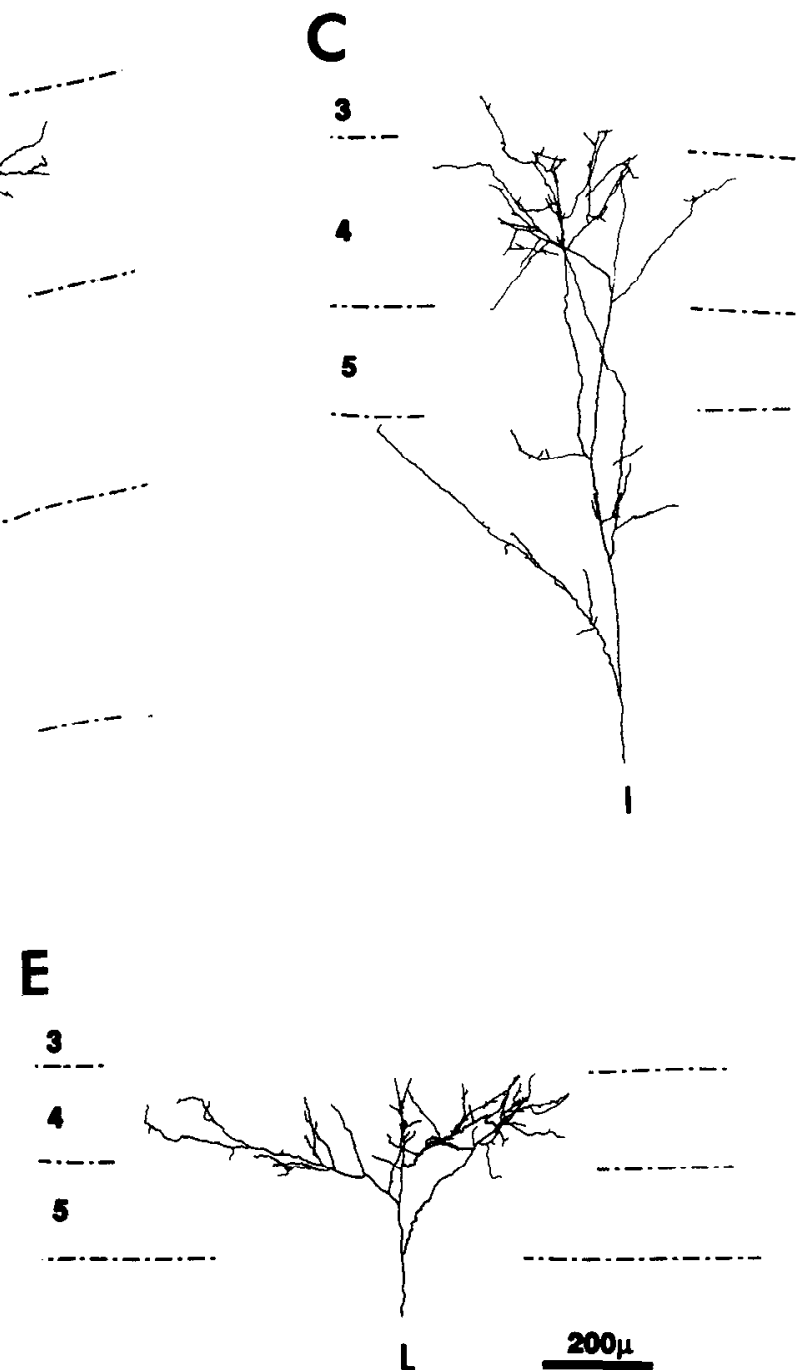

Figure 8. Camera lucida drawings of axons $K, J, I, M$, and $L(A-E$, respectively) reconstructed from area 17 of a P39 kitten (see Table 2). All axons are viewed from the coronal plane, the ventrodorsal axis of the cortical layers runs right to left. The figure demonstrates the variability in the morphology and pattern of arborization of axonal arbors. Arbors $K, J$, and $I$ project mainly to the upper tier of layer IV; arbors $M$ and $L$ ramify across the whole width of the layer.

(NN1, NN13, and NN14; Table 2). All arbors were located in the medial bank of the lateral gyrus and were confined well within area 17 , with the exception of arbor NN1 in which a farreaching branch extended into area 18.

Figure 8 shows the camera lucida drawings of the arbors reconstructed in two dimensions in the coronal plane. Arbors $\mathrm{K}$, $\mathrm{J}$, and $\mathrm{I}$ (Fig. $8 A-C$ ) ramified exclusively or predominantly in the upper portion of layer IV and the lower part of layer III. The terminal fields of the other two arbors, $\mathrm{M}$ and L (Fig. 8D,E), spanned the entire width of layer IV. Arbor K was unusual in that only a few short terminal ramifications were found in layer IV: two branches were located in different anteroposterior planes scparatcd by about $400 \mu \mathrm{m}$. In the coronal plane, arbors I and J covered a narrow strip of layer IV $(<700 \mu \mathrm{m})$. These arbors were also restricted along the anteroposterior axis, indicating that their terminal fields formed a compact and homogeneous patch of innervation.

In a few coronal sections through the lateral gyrus, the PHA-L labeling was intense enough to demonstrate ocular dominance patches. In this case, even though all labeled cells in the LGN were confined to layer A, ensuring that both the patches and all labeled axons served the same eye, the axonal trunk of arbor $L$ (Fig. $8 E$ ) penetrated into layer IV not in correspondence with, but between, two ocular dominance patches. The main axonal trunk of arbor $\mathrm{L}$ then bifurcated and directed its principal branches within the ocular dominance patches, as shown in the photomicrograph of Figure 9.

A more precise indication of the axonal morphology at this age is provided by the three arbors three-dimensionally reconstructed and illustrated in frontal and surface views (Fig. 10). When viewed in the coronal plane (Fig. 10A), the terminal arborization of both axons NN13 and NN14 each appeared as single dense patches in the upper tier of layer IV. In both axons, a few solitary branches from each patch of terminals extended in layer IV or crossed the layer III/IV border to invade layer III. In the coronal view, axon NN1 presented a primary cluster of terminals, innervating predominantly the upper portion of layer IV, and long, poorly ramified branches running parallel to 


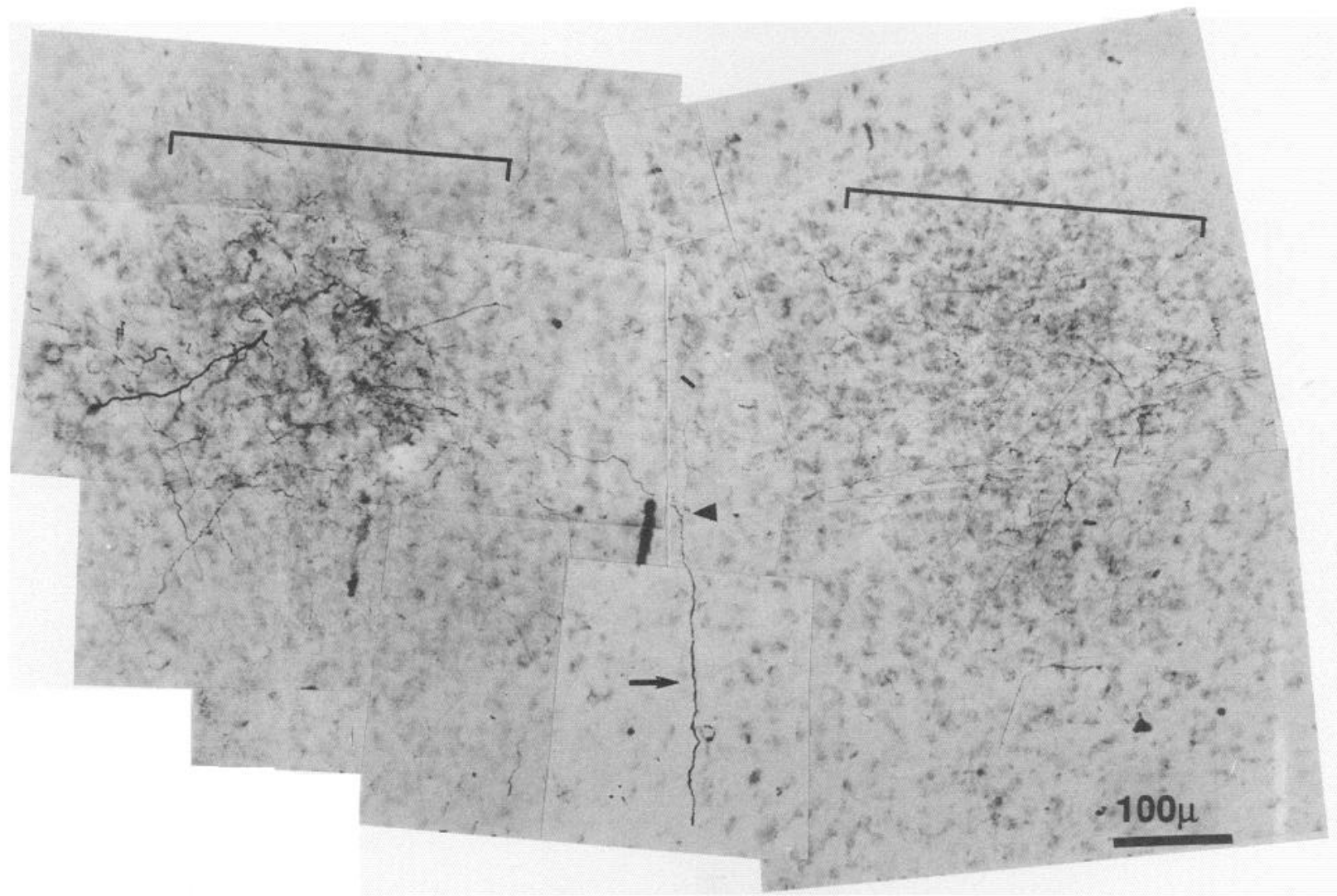

Figure 9. Photomicrograph of layer IV with the main parent axon trunk (indicated by an arrow) and initial collaterals (arrowhead) of axon L, the camera lucida drawing of which is illustrated in Figure 8. The photograph is a montage of several photomicrographs taken from different sections in series. The case illustrated is a P39 kitten in which the PHA-L injection site was entirely confined to lamina A of the LGN, so that both the axon and the general labeling in the cortex are ascribable to geniculocortical afferents serving the sole contralateral eye. Note the presence of two clumps of anterogradely labeled terminals (surmounted by brackets) identifiable as ocular dominance patches and the location, between, of the axon trunk of arbor L. The initial collaterals of the axon are directed toward the ocular dominance patches; moreover, the serial reconstruction of the entire arbor revealed that its terminal ramifications were predominantly located within the patches of eye-specific anterograde labeling.

the cortical layers in proximity to the layer III/IV border. Due to the representation of a three-dimensional arbor in a twodimensional plane, an axonal branch seems to recede toward the deeper cortical layers although it was in fact located in layer IV. When the three-dimensional reconstructions were rotated about the dorsal axis, a clear clustering in the terminal arborization became apparent in all three axons (Fig. 10B). Arbors NN13 and NN14 each demonstrated a single patch of dense innervation, while arbor NN1 formed two clumps of terminals separated by an axonal segment of about $200 \mu \mathrm{m}$ completely devoid of collaterals.

\section{Development of geniculocortical arbors in TTX-treated animals}

$P 23$. Four arbors were reconstructed from case MUC 719 in which retinal activity was blocked for $6 \mathrm{~d}$, from P17 to P23, when the animal was perfused. All were located in the medial bank of the lateral gyrus.

Figure 11 illustrates coronal and surface views of typical arbors. Arbors TTX15 and TTX18 ramified predominantly in the upper portion of layer IV, whereas the terminal field of TTX17 spanned the entire width of layer IV. The three arbors were spatially homogeneous and lacked a patchy organization, similar to arbors reconstructed at P19 and P23 in normal animals. In contrast to the arbors found in age-matched controls shown in
Figure 5, arbors in TTX-treated animals appeared more richly ramified. A quantitative analysis of the differences between these groups is presented in the last section of Results.

P29. Six axons were reconstructed from case MUC 714 (Table 1). In this animal, PHA-L injections were made into the LGN at P16 and retinal ganglion cell activity was continuously blocked for $12 \mathrm{~d}$, from P17 to P29, when the animal was perfused. Arbors were reconstructed from the medial bank of the lateral gyrus, all within area 17.

Figure 12 illustrates three of the six arbors reconstructed at P29, viewed in coronal (Fig. 12A) and surface (Fig. 12B) views. Two arbors (TTX1 and TTX6) innervated the upper portion of layer IV, while the terminal arborization of TTX2 was distributed throughout the entire width of layer IV. A few processes from all arbors rose to the layer III/IV border and extended into the lower portion of layer III. Two main features distinguished geniculocortical arbors in TTX-treated animals from agematched controls. First, the terminal arborization along the ventrodorsal axis was significantly broader in TTX-treated animals. Second, the terminal arborization of four out of six arbors reconstructed in the TTX-treated animals lacked the well-defined patchy terminal organization evident in age-matched controls (Fig. 6). The arbors illustrated in coronal and surface views in Figure 12, as well as arbor TTX5 (not illustrated), were homogeneously distributed throughout the entire cortical region. $P 39$. Seven axons were reconstructed from two animals (MUC 
P39

A

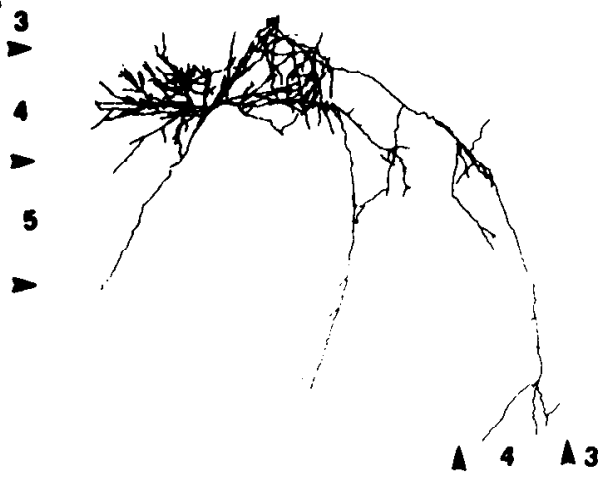

NN1

B

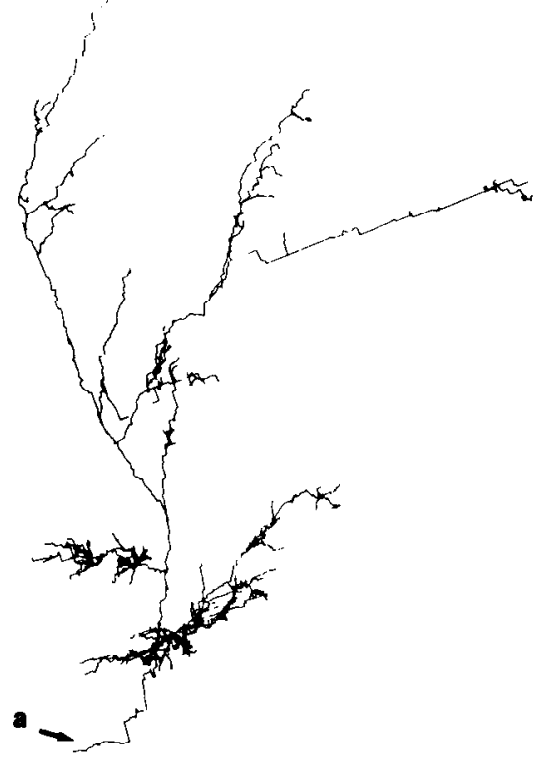

3

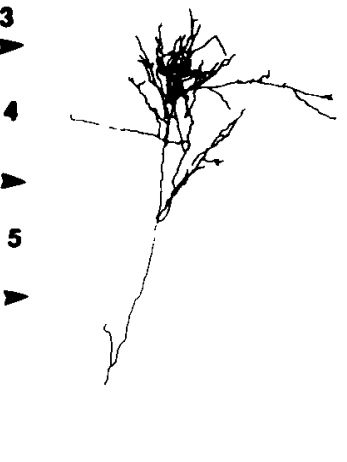

NN13

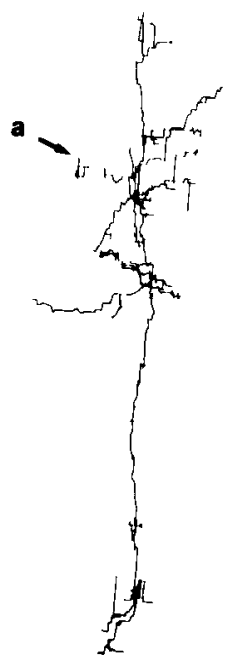

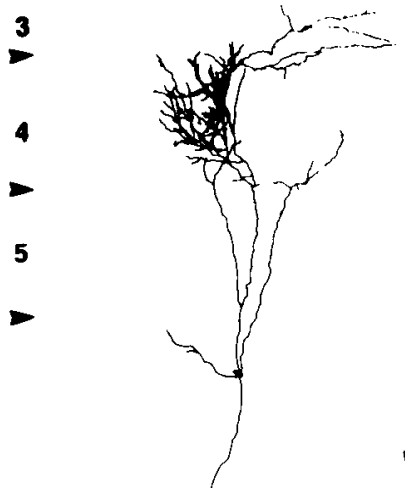

$V \longrightarrow D$

NN14

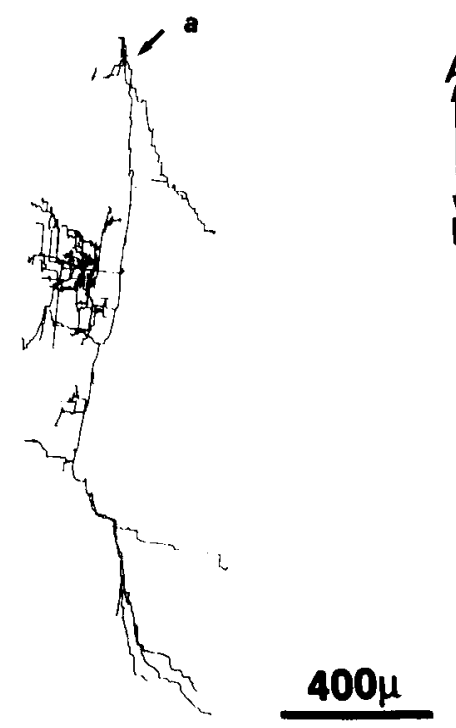

Figure 10. Axonal arbors $(N N 1, N N 13$, and $N N 14)$ reconstructed from kittens at P39. Axons are shown in coronal view $(A)$ and in surface view $(B)$. It is from this latter perspective that the terminal arborization in all three axons appears clearly segregated into one or two patches. In $B, a$ indicates the main axonal trunk. The scale bar applies to both $A$ and $B$.

720 and MUC 721, Table 1) in which PHA-L was injected at P30 and the retinal activity was blocked during two periods, from P13 to P26 and from P31 to perfusion at P39. Coronal and surface views of four arbors are illustrated in Figures 13 and 14. Arbors TTX7 and TTX11 appeared to bc organized in a circular fashion, while arbors TTX9, TTX10 (Fig. 14), and TTX12 (not illustrated) were poorly ramified, with smooth branches and few visible boutons.

\section{Apparently patchy arbors in TTX-treated animals}

At all ages, a few arbors appeared, at least by eye, to have a patchy organization. We have collected all such cases in Figure 15. Arbor TTX16, reconstructed at $\mathrm{P} 23$, ramifies predominantly in the upper portion of layer IV and appeared to be subdivided into two patches. A vague clustering pattern of the terminal arborization is also present in arbors TTX3 and TTX4, reconstructed at P29. Arbor TTX3 bifurcated in the upper part of layer $\mathrm{V}$ and each branch gave rise to two poorly demarcated patches of terminals in layer IV. The terminal arborization of arbor TTX4 appeared to form an elongated cluster of collaterals. Two arbors reconstructed at P39 (TTX8 and TTX13) could also be viewed as showing a patchy terminal organization. Arbor TTX8 was very elongatcd along the ventrodorsal axis, and also formed, along this same axis, an uninterrupted arborization spanning more than $800 \mu \mathrm{m}$ of layer IV. However, a small patch of terminals detached from the main arborization is clearly distinguishable. The patchy organization of TTX13 becomes apparent from the surface view of the arbor (Fig. 15B); however, note that the two patches are not fully separated. In animals at P23 and P39, although PHA-L injection sites were confined to layer A, no hint of patches or columnar segregation of the bulk labeling of geniculocortical afferents was evident. This finding was in contrast to the patchy appearance in the distribution of geniculocortical afferents in the normal animal at P39 (see Fig. 9, MUC 672). The quantitative analysis of these patches is presented below. 
P23

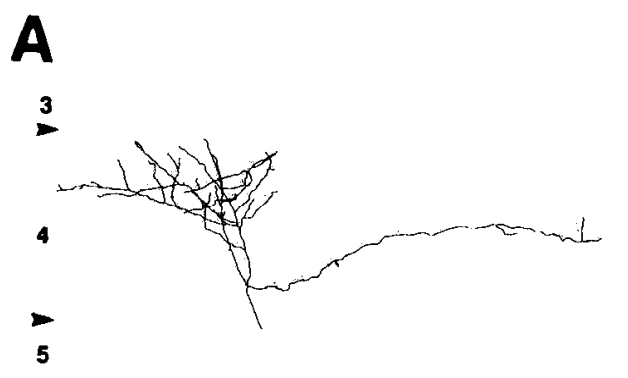

5

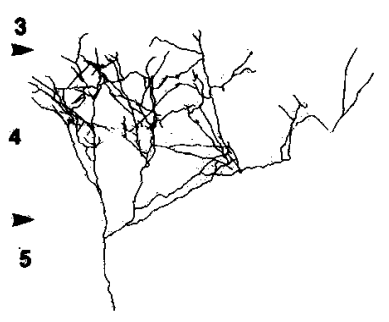

TTX17

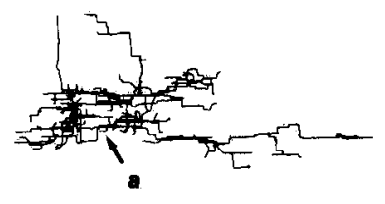

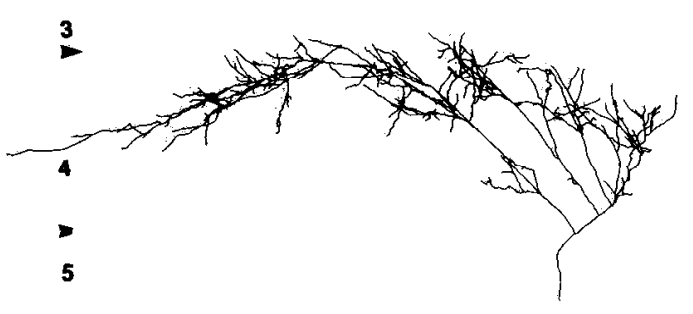
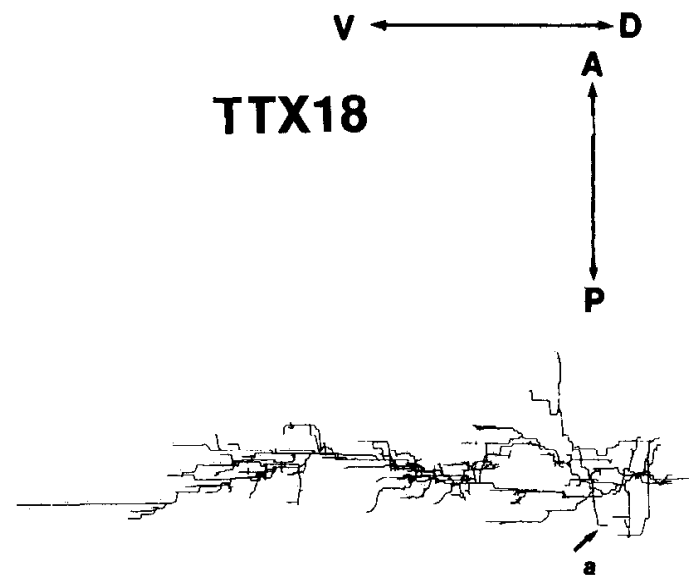

$400 \mu$

Figure 11. Computer reconstruction of PHA-L-immunostained axonal arbors (TTX15, TTX17, and TTX18) in area 17 of a P23 kitten in which retinal activity has been blocked by intraocular injections of TTX. Row $A$ shows the arbors in coronal view; row $B$ shows the same axons from the pial surface. In $B, a$ indicates the main axonal trunk as it enters into the gray matter. The scale bar applies to both both $A$ and $B$.

\section{Quantitative analysis of arbors in normal and $T T X$-treated animals}

The maturation of geniculocortical afferents during normal development appears to involve two major morphological changes of the terminal arborization. The first is a general enrichment and elaboration of the collateral branches in layer IV (compare Figs. 4 and 5 with Figs. 6 and 10). A second change is the transformation of the terminal field, initially homogeneous, into distinct clusters of terminals. In comparison, TTX-treated animals demonstrate a broader extension of the terminal arborization along the ventrodorsal axis, and most lack the characteristic patchy distribution of terminals in layer IV. In the following paragraphs, we provide a quantitative analysis of several parameters of the normal and TTX-treated groups at three different ages. For each arbor we measured (1) the VD extension of the arborization, (2) the coverage area of the terminal field in layer IV, (3) the total length, (4) the number of branch points, and (5) the density of the terminal field (see Materials and Methods). To characterize the patches of collaterals quantitatively, we defined the characteristics of a standard patch (with a procedure that will be explained below) and then assessed whether or not each arbor bears patches, the area of the patches, and the density of innervation inside the patch. With the exception of the VD extension measurements, which were performed in all arbors, all the other parameters were obtained only from arbors that were computer reconstructed. Unless otherwise specified, all statistical comparisons were calculated using the MannWhitney $U$ test.

\section{Ventrodorsal extension}

The extension of the terminal arborization along the ventrodorsal axis of the lateral gyrus may indicate the topographic precision of the geniculocortical map. Figure $16 \mathrm{~A}$ shows the distribution of the VD extension of the terminal arborizations, and Table 3 provides the medians and values for statistically significant comparisons between normal and TTX-treated animals at different ages. Although at P30/31 and at P39 the majority of the arbors appeared to be distributed toward smaller VD extensions compared to arbors at P19 and P23, 3 of the 15 arbors were sufficiently extended to make the difference between the older and younger groups statistically insignificant. In the TTX-treated group, there was no tendency for VD extensions to change with age. Comparison between normal and TTXtreated groups matched for age showed only the distribution of VD extensions at P39 to be significantly different. At P23 and P29-P31 the two experimental groups did not differ significantly because of the same three normal arbors noted above, but the TTX population as a whole showed a greater VD extension than the normals. 


\section{P29}

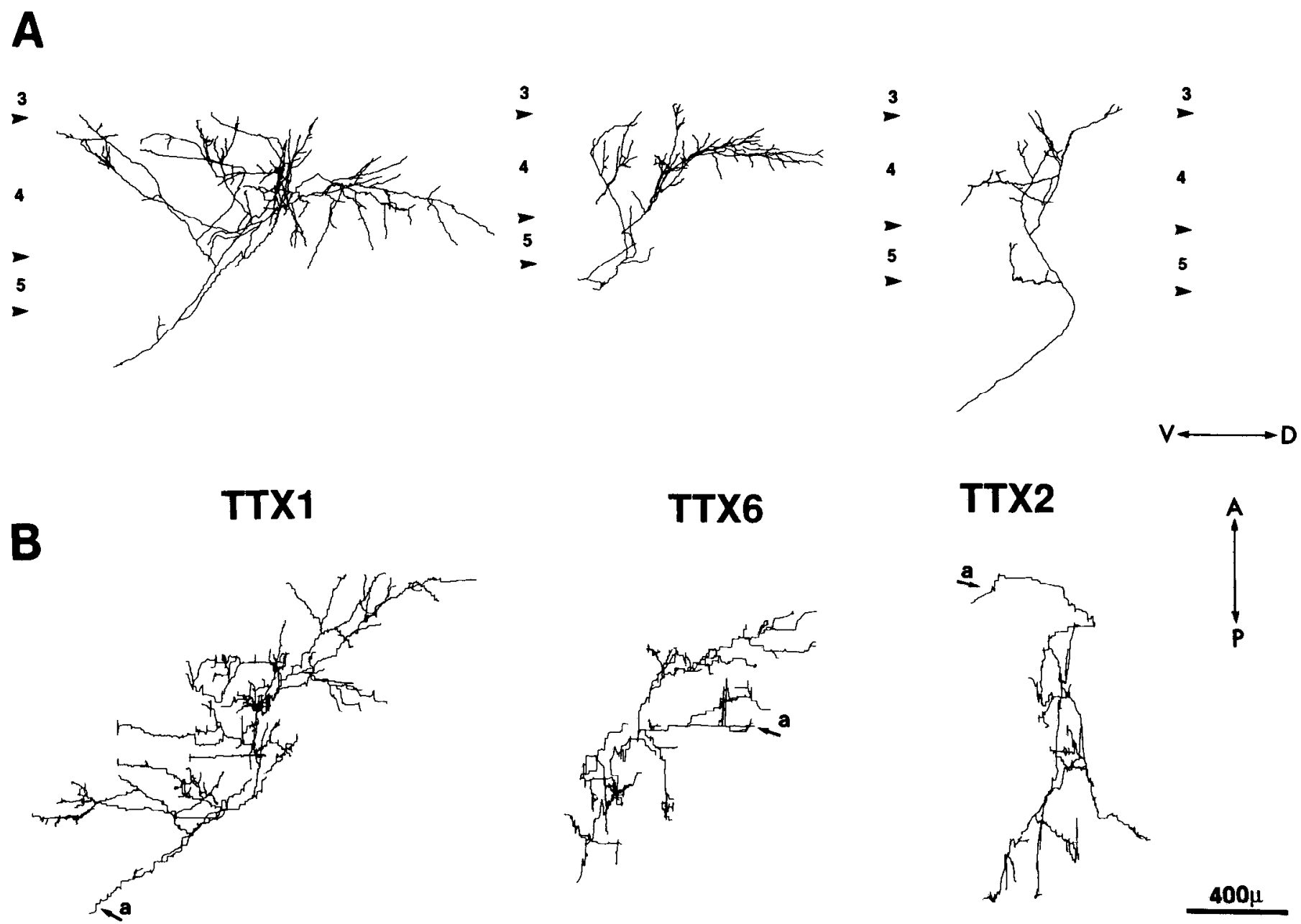

Figure 12. Axonal arbors (TTX1, TTX6, and TTX2) serially reconstructed from area 17 of a P29, TTX-treated kitten. Axons are shown in the coronal plane in $A$, and in surface view in $B$. Axons $T T X 1, T T X 6$, and $T T X 4$ arborize primarily in the upper tier of layer IV, whereas the terminal field of arbor TTX 2 spans the entire layer IV. The composite figure underlines the variability in morphology and ventrodorsal extent of the axonal arbors when viewed coronally, and the absence of a clear segregation of their terminal field in patches in both the frontal and surface views. In $B$, $a$ indicates the main axonal trunk as it enters into the gray matter. The scale bar applies to both $A$ and $B$.

If afferent arbors were to remain fixed in relation to the cortical cells, then the extension of the arborization should grow in a manner proportional to the growth of the cortex. We evaluated the linear growth of the cerebral hemispheres by measuring their perimeter, as viewed from the coronal plane. Although it is not known whether the different cortical areas have a different rate of developmental growth, it is reasonable to speculate that the linear expansion of the perimeter of the lateral gyrus provides a fair representation of the growth of area 17. The pial perimeter of the lateral gyrus, from the depth of the suprasplenial sulcus to the depth of the lateral sulcus, was measured in every second or third section ( $80 \mu \mathrm{m}$ per section). In order to assure that in all animals comparable portions of the lateral gyrus were selected, seven sections were chosen at an anteroposterior coronal level containing, at the subcortical level, the LGN. Specifically, the most posterior measurement was collected at an AP level corresponding to the posterior pole of the LGN. Figure $16 B$ illustrates, for both normal and TTX-treated groups, the means and SDs of the lateral gyrus perimeter at the different ages. In normal animals, no statistically significant differences were found between $\mathrm{P} 19$ and $\mathrm{P} 23$ or between $\mathrm{P} 30 / 31$ and $\mathrm{P} 39$, whereas all other differences were significant $(p<0.01)$. Among TTX-treated animals, all differences were significant $(p<0.01)$. In agematched comparisons, normal and TTX-treated groups were not statistically different.

To facilitate comparisons among the different ages and between the two experimental groups, the mean of the perimeter of the lateral gyrus obtained in each case can be expressed as a percentage of the mean found in normal animals at P39. Figure $16 \mathrm{C}$ illustrates, for both experimental groups and for all age groups, the VD extension normalized to the mean perimeter value of normals at P39. The comparisons within and between groups demonstrate the same statistical trends as those described for Figure 16A indicating that the changes of the VD extension are the same either in absolute terms or relative to the growth of the brain.

\section{Coverage area}

The measurements of the VD extension of the arbors presented above show that the area of the cortex over which the arbors 


\section{P39}
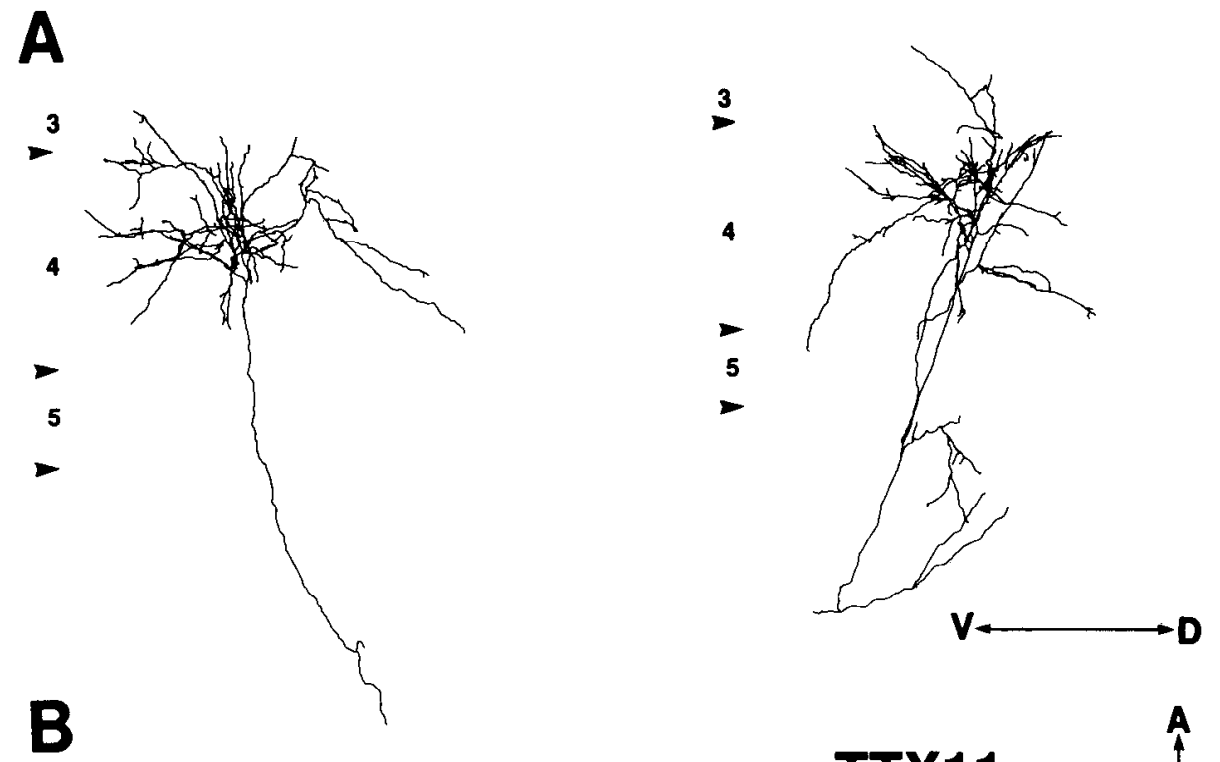

Figure 13. Axonal arbors (TTX7 and $T T X 11)$ serially reconstructed in area 17 of a P39 kitten raised with a TTXinduced retinal blockade. In row $A$, the axons are shown in coronal view; in $B$ they are shown as viewed from the pial surface. The two axons arborize in the upper portion of layer IV. In $B, a$ indicates the parent axonal trunk. The
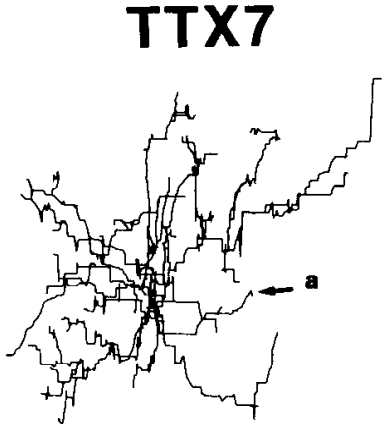

\section{TTX11}

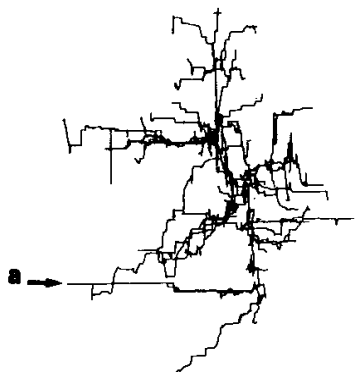
scale bar applies to both $A$ and $B$.

\section{P39 \\ A}

TTX9

Figure 14. Two unusual axonal arbors $(A, B)$ reconstructed in area 17 of a TTX-treated P39 kitten. Both axons have their terminal field in the upper tier of layer IV; their arborizations are formed by very fine, smooth branches oriented ventrally relative to the position of the parent axonal trunk. 
The Journal of Neuroscience, August 1993, 13(8) 3563

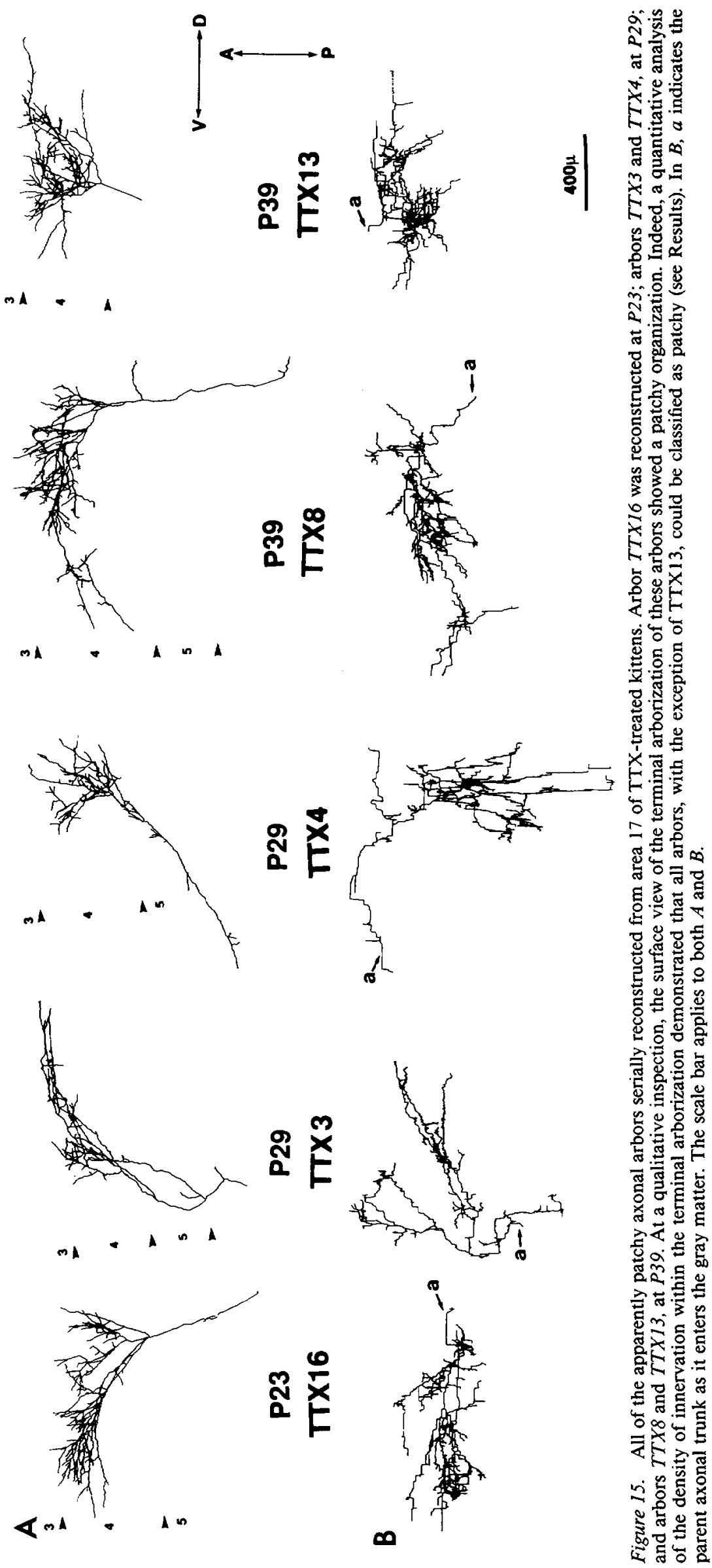



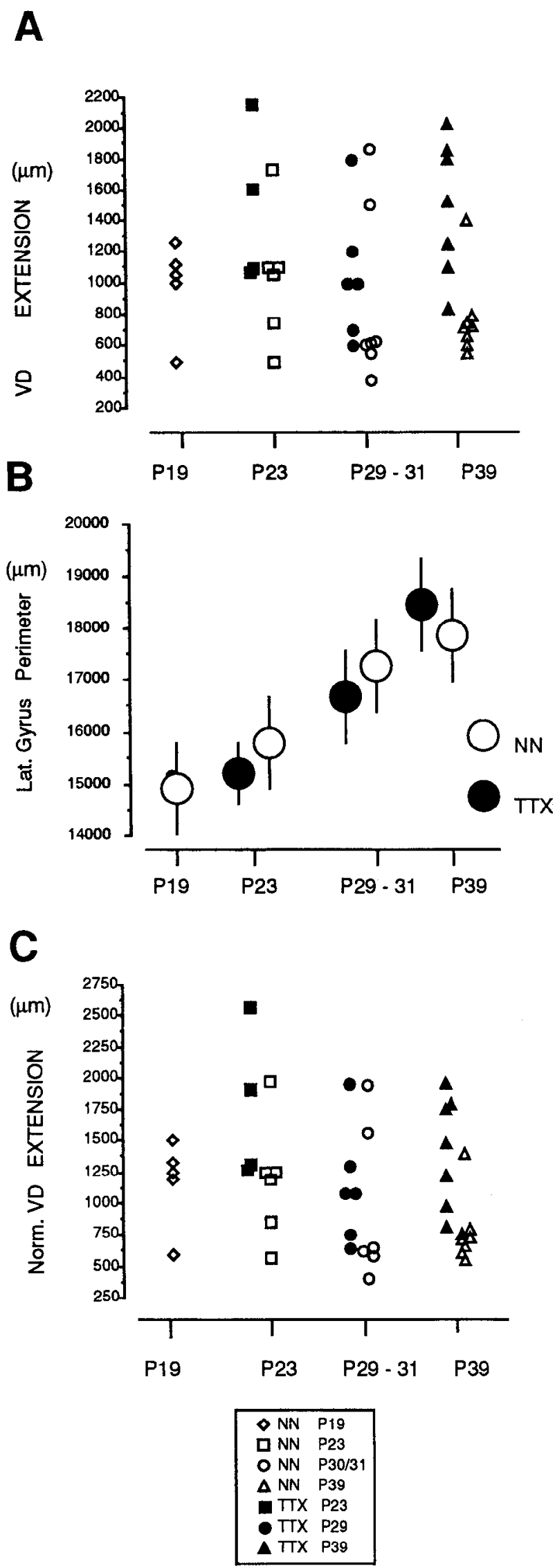

Figure 16. A, Scattergram of the extensions (ordinates, in micrometers) of the terminal arborizations along the ventrodorsal axis of layer IV. The data for normal (open symbols) and TTX-treated animals (solid

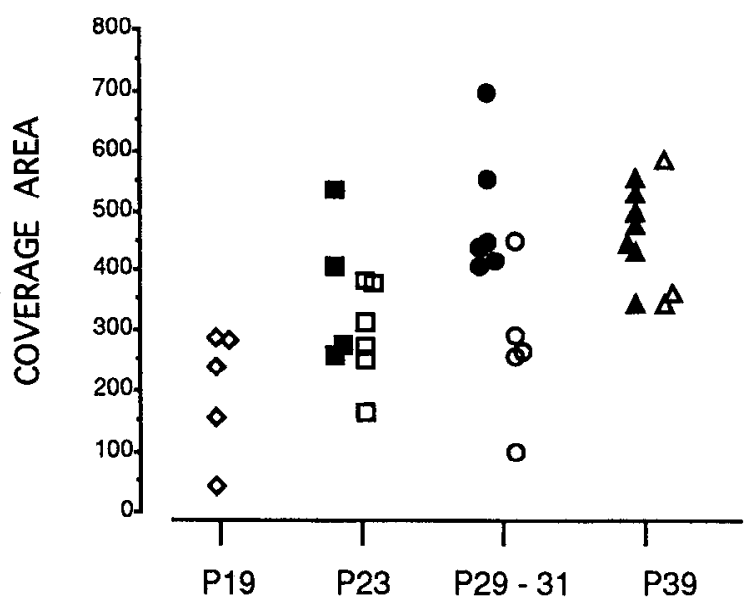

\begin{tabular}{|c|c|}
\hline$\diamond N N$ & P19 \\
\hline 口 NN & P23 \\
\hline O NN & P30/31 \\
\hline$\Delta N N$ & P39 \\
\hline$\pi \mathrm{T} X$ & P23 \\
\hline - TIX & P29 \\
\hline$\Delta \pi X$ & P39 \\
\hline
\end{tabular}

Figure 17. Scattergram of the surface area of arbors expressed in 1000 $\mu \mathrm{m}^{2}$ (ordinate). The data for normal (open symbols) and TTX-treated animals (solid symbols) are subdivided in age groups (abscissa).

ramify decreased substantially from P19-P23 to P29-P39 during normal development. However, the arbors in the younger animals are generally sparse and do not branch to fill most of the cortical area over which they extend. We computed a measure of the cortical coverage area actually occupied by the arbor, by measuring the area of layer IV in surface view that was within $50 \mu \mathrm{m}$ from any branch of the arbor (see Materials and Methods for details). This measure would be expected to increase if the arbors ramified to fill the territory over which they had extended more sparsely at an earlier age. Figure 17 shows the distribution of the coverage areas of the arbors, while Table 3 provides the medians and values for statistically significant comparisons between normal and TTX-treated animals at different ages. No differences were found among the P19, P23, and P30/31 age groups; however, at $\mathrm{P} 39$ arbor areas were significantly larger compared to P19 and P23. Arbor areas were not significantly different between the ages of P39 and P30/31. Taken together, these results indicate that there is a progressive increase of arbor coverage area between $\mathrm{P} 23$ and P39, with arbors at P30/31 presenting the greatest variability in size. In the TTX-treated animals, arbor area did not vary with age. A comparison between normal and age-matched TTX-treated animals showed a significant difference only in the P29-P31 age group; however, when the data at the different ages were combined, the TTXtreated group, considered as a wholc, demonstrated a significantly greater arbor area compared to the normal group.

symbols) are subdivided in age groups (abscissas). B, Mean and SD of the perimeter of the lateral gyrus measured at the different ages, in both normal and TTX-treated animals. $C$, Normalized VD extension of the terminal arborization. This scattergram has been obtained from the scattergram in $A$, by normalizing each value to the mean perimeter value of normal animals at P39. 


\begin{tabular}{|c|c|c|c|c|c|c|c|c|c|c|c|c|c|c|c|c|c|}
\hline & \multicolumn{3}{|c|}{$\begin{array}{l}\text { VD extension } \\
(\mu \mathrm{m})\end{array}$} & \multicolumn{2}{|c|}{$\begin{array}{l}\text { Arbor surface } \\
\text { area }\left(10^{3} \mu \mathrm{m}^{2}\right)\end{array}$} & \multicolumn{3}{|c|}{$\begin{array}{l}\text { Arbor total } \\
\text { length }(\mu \mathrm{m})\end{array}$} & \multicolumn{3}{|c|}{$\begin{array}{l}\text { Number of } \\
\text { branch points }\end{array}$} & \multicolumn{3}{|c|}{$\begin{array}{l}\text { Average branch } \\
\text { length }(\mu \mathrm{m})\end{array}$} & \multicolumn{3}{|c|}{$\begin{array}{l}\text { Arbor density } \\
\left(\mu \mathrm{m} / 1000 \mu \mathrm{m}^{2}\right)\end{array}$} \\
\hline & $\mathrm{N}$ & & TTX & $\mathrm{N}$ & TTX & $\mathrm{N}$ & & TTX & $\mathrm{N}$ & & TTX & $\mathrm{N}$ & & TTX & $\mathbf{N}$ & & TTX \\
\hline & & {$\left[{ }^{*}\right]$} & & & & & {$[*]$} & & & & & & & & & & \\
\hline P19 & 1050 & & & (1) 240 & & 9656.9 & & & (1) 40 & & & 276.3 & & & 8.4 & & \\
\hline $\mathrm{P} 23$ & 1075 & & 1355 & (2) 294 & 343 & $\begin{array}{c}7538.8 \\
*\end{array}$ & $*$ & 18157.2 & $\begin{array}{c}34.5 \\
* *\end{array}$ & $*$ & 105 & $\begin{array}{c}230.3 \\
* *\end{array}$ & & $\begin{array}{c}171.8 \\
*\end{array}$ & $\begin{array}{r}8.4 \\
* *\end{array}$ & $* *$ & 14.2 \\
\hline P29-P31 & 620 & & 1000 & $267^{*}$ & 447 & 12848.6 & & 15807.8 & $\begin{array}{c}96 \\
w /(1)^{* *} \\
w /(2)^{* *}\end{array}$ & & 73 & 133.8 & $* *$ & 252.9 & 14.4 & & 11.6 \\
\hline P39 & 718 & $* *$ & 1520 & $\begin{array}{r}364 \\
w /(1)^{* *} \\
w /(2)^{*}\end{array}$ & 474 & 10923.1 & & 16843.7 & $\begin{array}{c}99 \\
w /(1)^{*} \\
w /(2)^{* *}\end{array}$ & & 83 & 154.7 & & 202.9 & 13 & & 11.7 \\
\hline
\end{tabular}

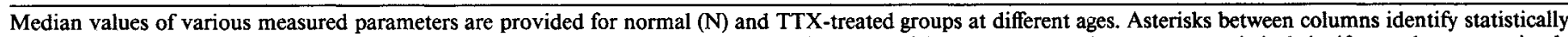

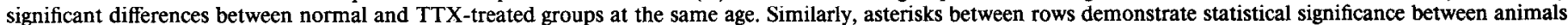

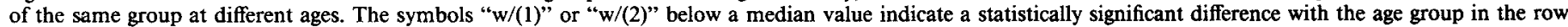

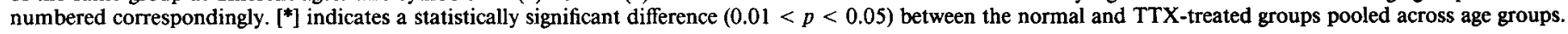

$* 0.01<p<0.05$.

** $p<0.01$.

\section{Length and branch points of the terminal arborization}

To analyze the progressive changes in geniculocortical arbors during development, we have measured the total length of all the branches of the terminal arborization within layer IV (see Materials and Methods). The scattergram in Figure $18 \mathrm{~A}$ shows the distribution of the total length of each arbor, while Table 3 provides the medians and values for statistically significant comparisons between normal and TTX-treated animals at different ages. In normal animals, the total length of the terminal arborizations did not vary significantly between $\mathrm{P} 19$ and $\mathrm{P} 23$, and between P30/31 and P39. It was, however, significantly smaller at P23 than at P30/31, and showed a tendency, though not significant, to be smaller at P23 than at P39 $(p=0.08)$. The TTX-treated group was characterized, at all ages, by a great variability in the total length of the terminal arborization. The length distributions did not differ significantly among the three age groups. In comparing normal and TTX-treated animals, two results are worth noting. First, the TTX-treated group had at all ages the greatest values of total lengths. Second, the TTX group as a whole showed a significantly greater length of the terminal arborization compared to the normal population, although in age-matched comparisons, a significant difference between the two experimental groups was present only at P23.

Assuming that the total length of the terminal arborization provided a general indication of the overall growth of an axon, then the number of branch points present in the terminal field should provide a clearer measure of the complexity of the arborization and the degree of ramification. The distribution of the number of branch points for each geniculocortical arbor is plotted in Figure $18 B$, while Table 3 provides the medians and values for statistically significant comparisons between normal and TTX-treated animals at different ages. In normal animals, while no significant difference was found in the degree of branching between the ages of P19 and P23, and between the ages of P30/31 and P39, the number of branch points showed a significant increase with age from $\mathrm{P} 19$ to $\mathrm{P} 30 / 31$ and to P39, and from P23 to P30/31 and to P39. In the TTX-treated animals, no significant differences were found across these age groups. When age-matched, normal and TTX-treated animals were compared, a significant difference was evident only at the youngest age. In contrast, no significant differences were demonstrated between the older experimental groups at P30/31 and P39, or between normal and TTX-treated arbor populations. It should be noted that the TTX-treated group, particularly at P23 and P39, showed a much greater variability in the number of branch points than the normal group. A similar scatter has been already described for the distribution of the arbor total length (Fig. 18A).

The ratio between the two parameters, total length and number of branch points, provides an estimate of the average branch length and, as such, an indication of the complexity and spatial restriction of the arbor within layer IV. The scattergram of Figure $18 \mathrm{C}$ shows the distribution of the average branch length, and Table 3 provides the medians and values for statistically significant comparisons between normal and TTX-treated animals at different ages. Two main observations emerge from Figure $18 C$. First, in normal animals there is a dramatic decrease with age in the branch length. Second, at all ages, the TTXtreated animals demonstrated great variability in average branch length, suggesting that age does not produce a developmental change in this parameter. In normal animals, the average branch length is significantly greater at P19 and at P23 than at older ages. For the TTX-treated group, a statistically significant difference was found only between the ages of P23 and P29. Again, in an age-matched comparison between normal and TTX-treated animals, the distributions of the average branch length were significantly different only at P29-P31. However, when the data at all ages were combined, the two experimental groups did not differ significantly.

\section{Density}

Another way to quantify the degree of elaboration and ramification of the arbors is the evaluation of the mean density of the terminal arborization (see Materials and Methods). The maturational changes in mean density are shown in the scattergram of Figure 19A, while Table 3 provides the medians and values for statistically significant comparisons between normal and TTXtreated animals at different ages. Normal animals showed a clear age-dependent increase in the mean density of the terminal arborization. The increase in density appears to occur abruptly 
A

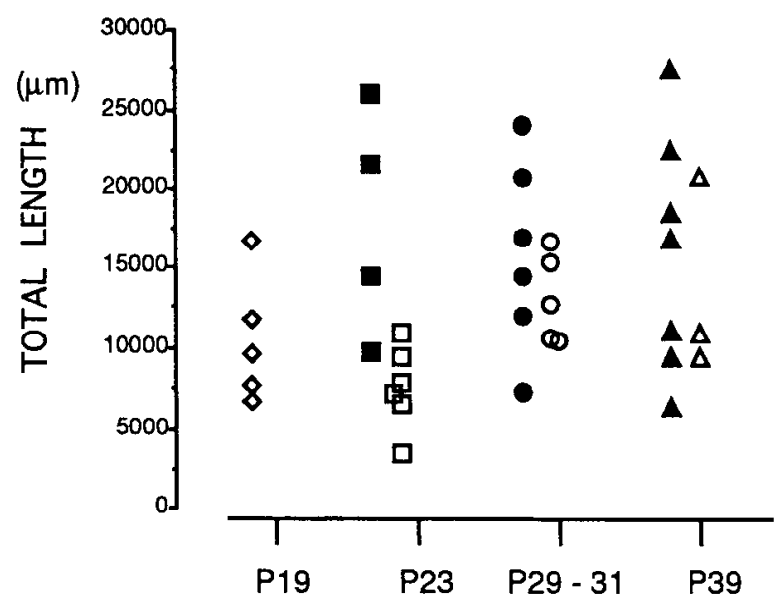

B
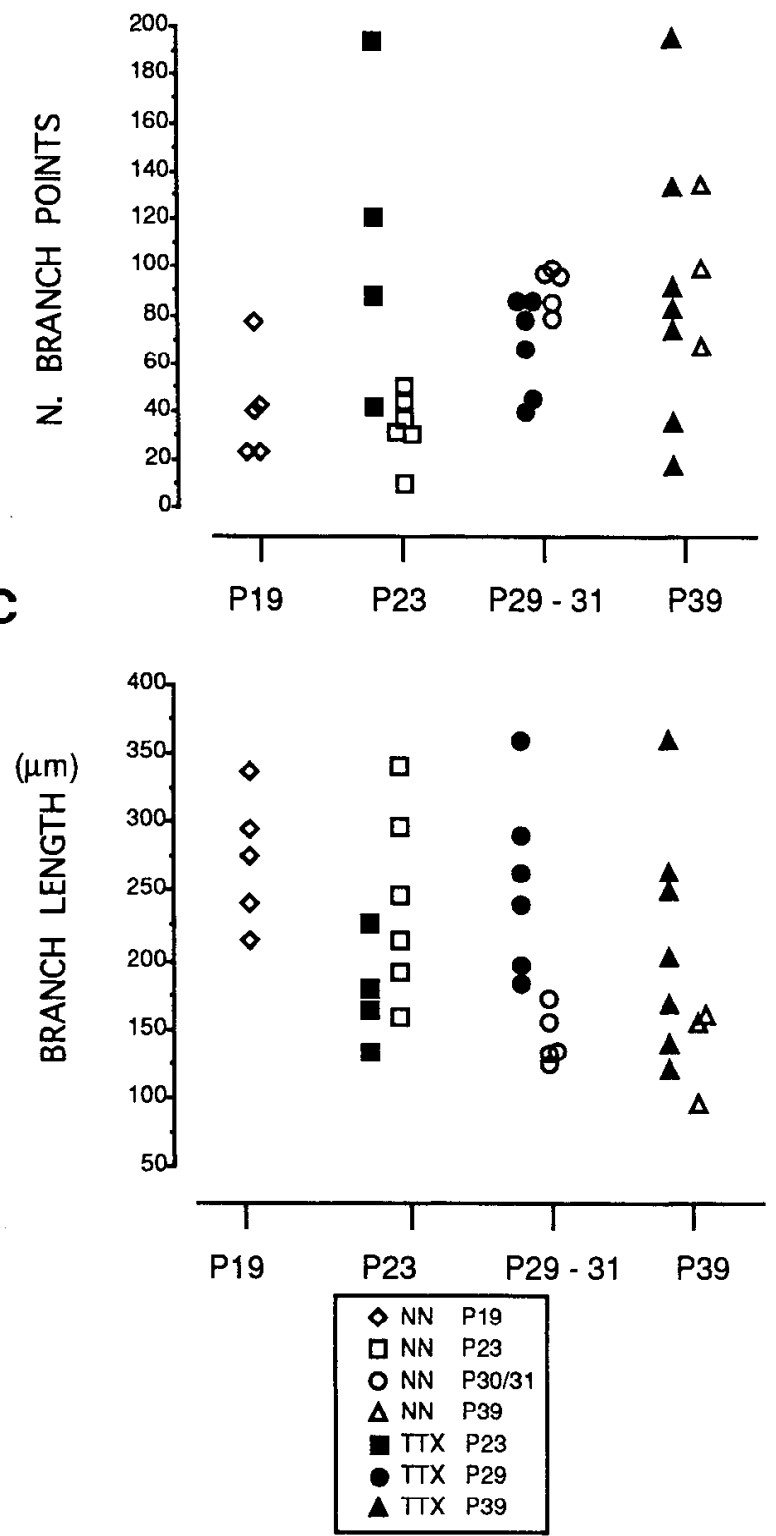

Figure 18. Analysis of terminal arborization of geniculocortical afferents in layer IV. $A$, Scattergram of the total lengths (in micrometers) of the terminal arborizations. $B$, Scattergram of the number of branch
A

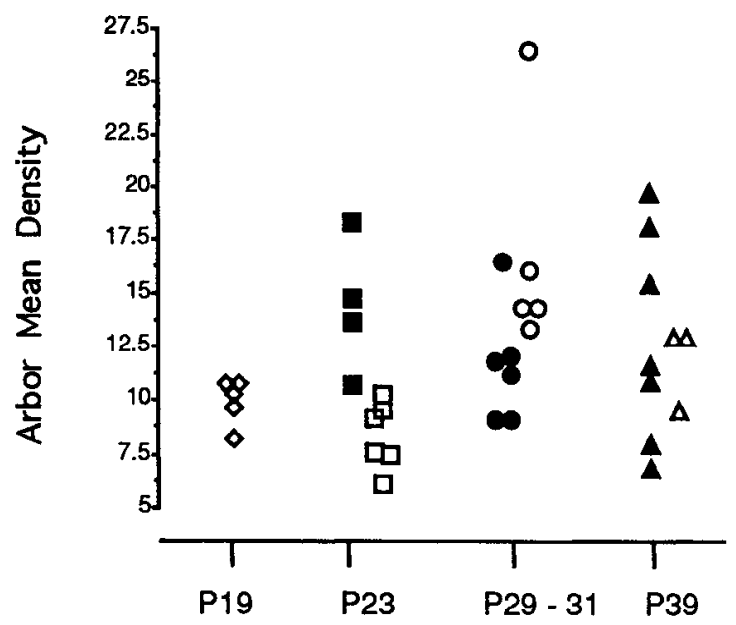

B
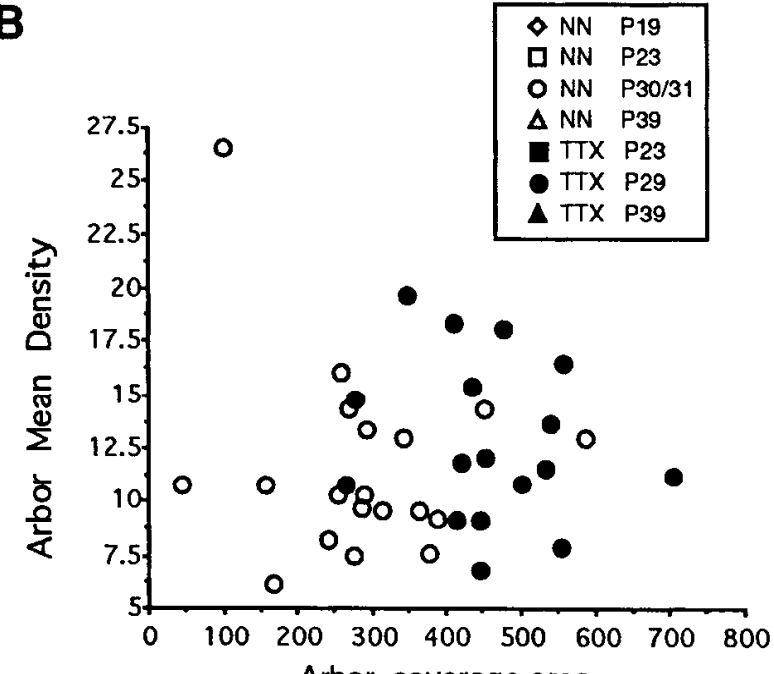

Arbor coverage area

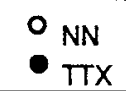

Figure 19. Analysis of the density (expressed in $\mu \mathrm{m} / 1000 \mu \mathrm{m}^{2}$ ) of the terminal arborization in layer IV. $A$, Scattergram of the mean density across the terminal arborization of geniculocortical axons. The data for normal (open symbols) and TTX-treated animals (solid symbols) are subdivided in age groups (abscissas). B. Distribution of the arbor mean density relative to the arbor surface area (expressed in $1000 \mu \mathrm{m}^{2}$ ) for the normal (open symbols) and TTX-treated animals (solid symbols).

between P23 and P30/31, when the median density increased significantly from 8.4 to $14.4 \mu \mathrm{m} / 1000 \mu \mathrm{m}^{2}$. Note the exceptionally high density of one arbor corresponding to NN2 at P30/ 31 (Fig. 6), which was characterized by a very small and richly ramified terminal arborization. No significant differences were found between P23 and P19 and between P30/31 and P39.

points. $C$, Scattergram of the average branch length (in micrometers) of the terminal arborizations corresponding to the ratio between the total length and the number of branch points. The data for normal (open symbols) and TTX-treated animals (solid symbols) are subdivided in age groups (abscissas). 
In the TTX-treated group, the arbor mean density was highly variable and did not appear to change with age. In a comparison with the group of normal animals, a noteworthy difference was found at P23 when TTX arbors showed a higher density than their age-matched normal counterparts. Yet, when pooling all ages, the two experimental groups did not differ significantly in the distribution of arbor mean density. This outcome is also evident from the scattergram in Figure 19B, which shows the relationship between the mean arbor density and arbor coverage area for the two experimental groups. Two conclusions can be drawn from this figure. First, normal and TTX arbors have similar ranges of mean density values. Second, for similar density values, arbors in TTX-treated animals have significantly larger areas $(p<0.05)$.

\section{Organization of the terminal arborization of geniculocortical afferents}

In single axon reconstructions, the patchy organization of an axon terminal arborization indicates that segregation into eyespecific domains has occurred. Assessing this organization is difficult, particularly in arbors that did not show clearly defined clumps of terminals upon qualitative inspection. To define whether a terminal arborization could be considered subdivided into patches, we performed a quantitative evaluation of both the fluctuation and distribution of the density of collateral branches within the terminal arborization.

The first analysis was aimed at demonstrating the zones of relatively high density of innervation within each terminal arborization. The highest density value and a threshold density, defined as half-maximum density, were computed for each arbor. These two density values delineate high-density zones within each arbor. Figure $20 \mathrm{~A}$ illustrates the distribution of the maximal densitics within these high-density zones, in normal and TTX-treated animals subdivided by age. Three main findings are evident. First, in normal animals, there is a substantial increase in the maximal densities of the high-density zones between P30/31 and P39 compared to the younger ages (comparison between $\mathrm{P} 19$ and $\mathrm{P} 30 / 31$ or $\mathrm{P} 39: p<0.005$ and $p=0.005$, respectively; comparison between $\mathrm{P} 23$ and $\mathrm{P} 30 / 31$ or $\mathrm{P} 39: p=$ 0.0001 and $p=0.01$, respectively). This result was expected since the mean density of the total arbors showed a similar maturational course. Second, no significant differences were found in the distribution of densities among the TTX-treated groups at different ages. Third, at P29-P31 and P39, TTX and normal arbors had similar distributions of maximal densities.

Figure 20. A, Analysis of the high-density zones within the terminal arborization. For each arbor, the highest density value (expressed in $\mu \mathrm{m} / 1000 \mu \mathrm{m}^{2}$ ) was determined, and a threshold density was defined as equaling half of the value of the maximal density. The scattergram in $A$ shows the distribution of the maximal densities within these highdensity zones. $B$, Distribution of areas of the standard patches (expressed in $1000 \mu \mathrm{m}^{2}$ ). The quantitative definition of a standard patch of terminals is described in Results. $C$, Decrease in density of the terminal arborization in the interpatch space. The density of the interpatch space was calculated at the midpoint between the two patches. However, for pairs of patches separated by more than $300 \mu \mathrm{m}$ (average distance between patches in normal animals), and for single patches, the density of the interpatch space was calculated at $150 \mu \mathrm{m}$ from the patch border. The density at the interpatch space is expressed as percentage difference from the minimum density of the standard patch $\left(38 \mu \mathrm{m} / 1000 \mu \mathrm{m}^{2}\right)$. In $A-C$, the data for normal (open symbols) and TTX-treated animals (solid symbols) are subdivided in age groups (abscissas).

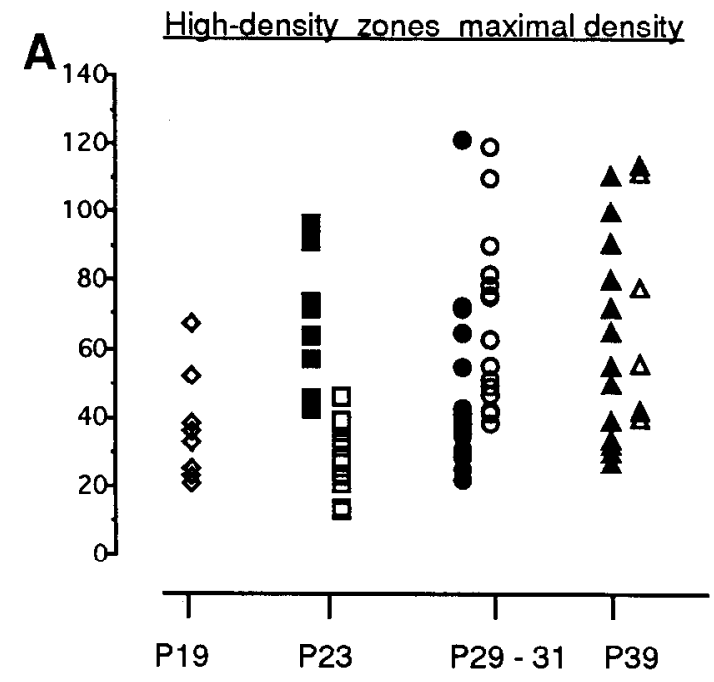

$B \quad 70$ Standard patch area

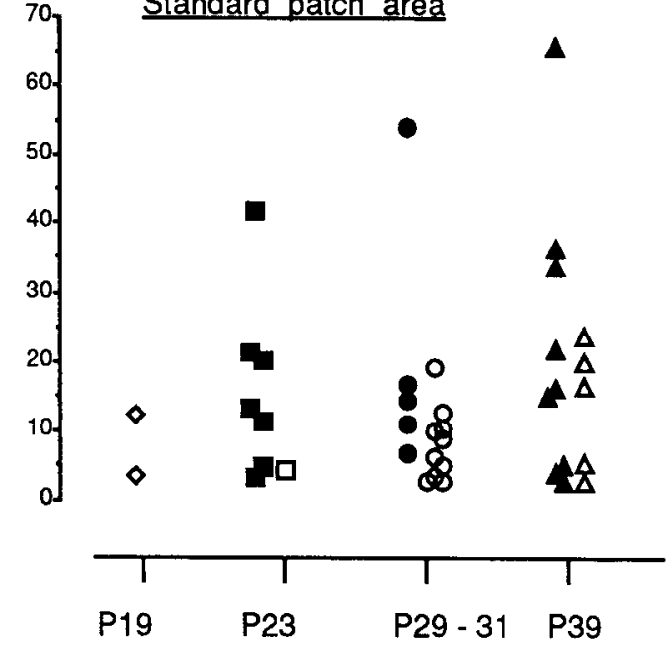

Percent decrease in density in the interpatch zones

C

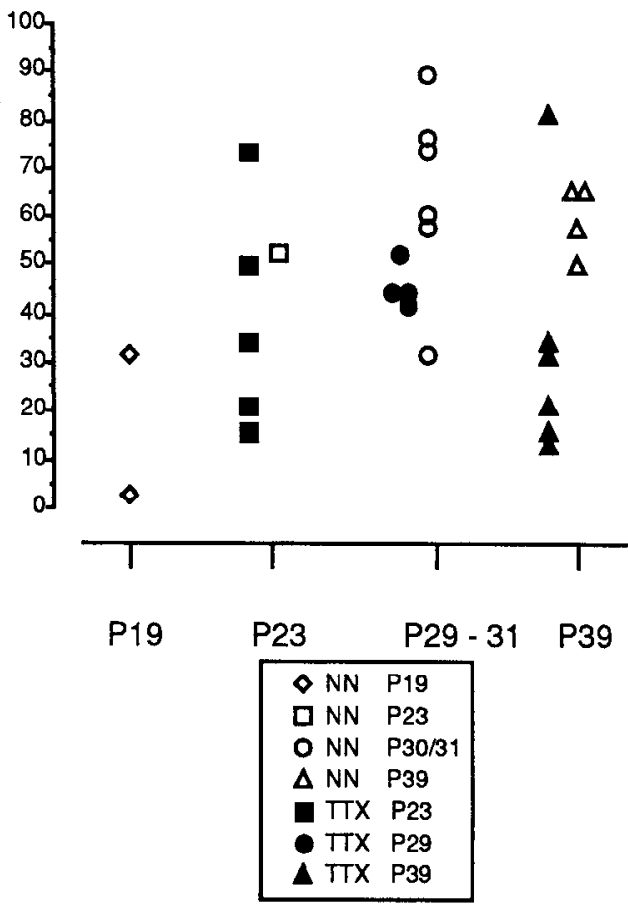


Figure 21. Density distributions of four arbors viewed from the pial surface. Two arbors above were reconstructed in normal animals at $P 30$ and $P 39$, respectively, and two below were reconstructed in TTX-treated animals at P39. The same arbors were shown in Figures 6, 10, and 13. The dark, outlined areas correspond to zones in which the display threshold was set for a minimum density of $38 \mu \mathrm{m} / 1000 \mu \mathrm{m}^{2}$. A "standard patch," as defined in Results, must meet two criteria. First, the minimum density must exceed $38 \mu \mathrm{m} / 1000$ $\mu \mathrm{m}^{2}$, and second, if the borders of two patches were separated by less than 90 $\mu \mathrm{m}$, they were considered as a single patch. For example, in arbor $N N 10$, four patches are outlined on the basis of thresholding alone, but with the additional criterion of $90 \mu \mathrm{m}$ separation, the arbor was considered to have only two patches. Also note that for TTX-treated animals, a large area surrounding the patch is of relatively high density, in contrast to normals where the entire area surrounding a patch is of a markedly reduced density. Scale bar, $200 \mu \mathrm{m}$.

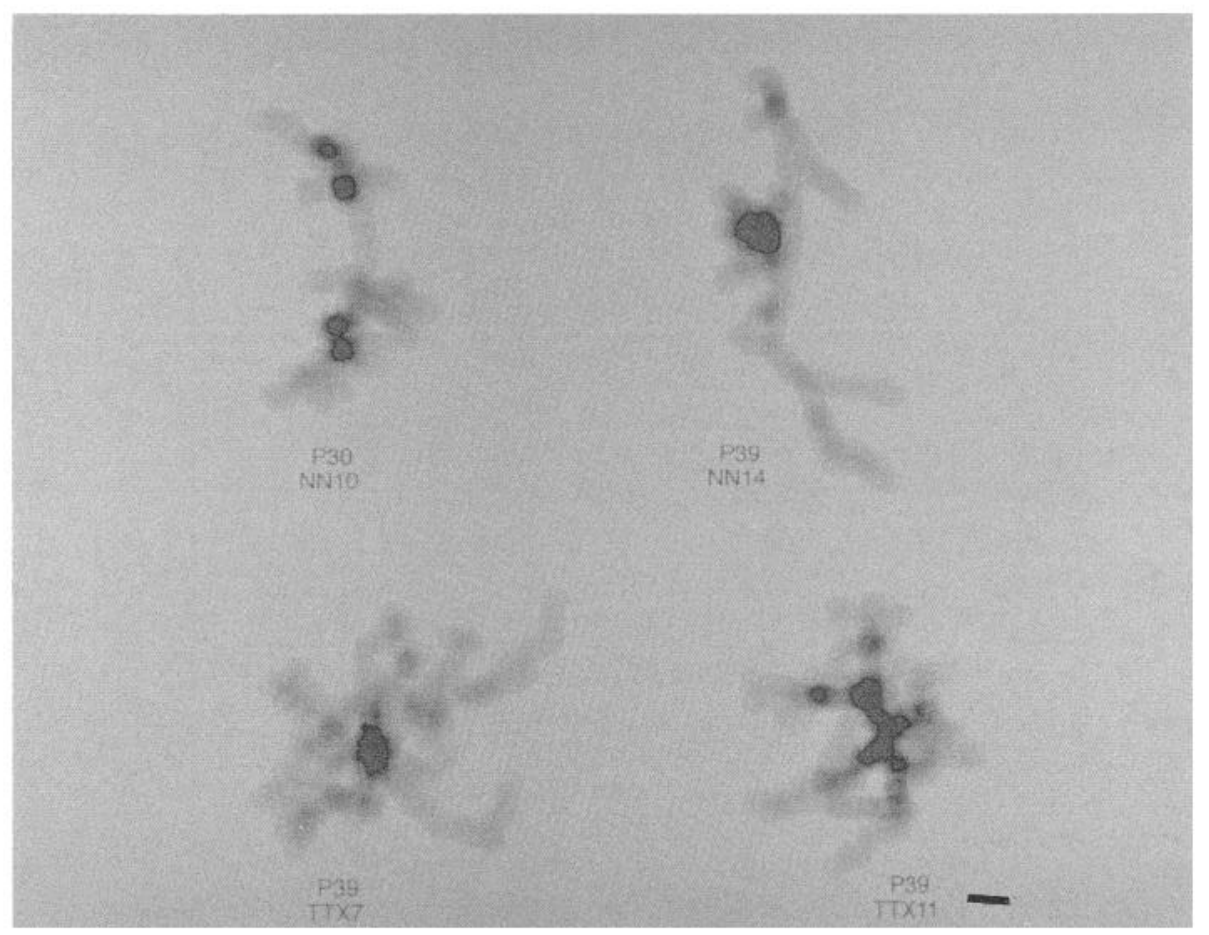

This type of analysis indicates that, both in normal and in TTX-treated kittens, the arbors are not uniform. To understand whether nonuniformities in the arbors were consistent with ocular dominance patches, we further analyzed the areas of highdensity patches in the arbor (Fig. 20B) and the reduction in arbor density in the space between patches (Fig. 20C). Because each arbor is considered independently, the analysis above does not allow a direct comparison of the characteristics of the highdensity zones. Similarly, it does not allow for an absolute evaluation of the degree of segregation. We were thus motivated to find a quantitative definition of a standard high-density zone that would be applicable in both experimental groups and at all ages.

Because the segregation of afferents, as demonstrated by autoradiography, is well advanced by P30 and almost completed by P39 (LeVay et al., 1978), we felt justified in defining the quantitative characteristics of a standard high-density zone, or standard patch, on the basis of patches found in normal animals at P30/31 and P39. Two constraints were used to identify a patch: a minimum density and a minimum distance from another patch. The standard minimum density, used for normalization and comparison within and across experimental groups, was obtained from the average threshold density at the border of patches at P30/31 and at P39. The arbors considered for this analysis were NN1-NN3 and NN10-NN14. The mean of the threshold densities of these arbors was $38 \mu \mathrm{m} / 1000 \mu \mathrm{m}^{2}$. The standard minimum distance between two individual patches was obtained from the smallest minimum distance separating two patches. Arbors NN1, NN10, and NN12 were selected for this analysis, as the pairs of patches were well circumscribed. The smallest of the minimum distances separating each pair of patches was $90 \mu \mathrm{m}$ (arbor NN1, Fig. $\cdot 10 B$ ). Thus, two patches, defined on the basis of the threshold standard density $(38 \mu \mathrm{m} /$ $1000 \mu \mathrm{m}^{2}$ ), located less than $90 \mu \mathrm{m}$ apart, were considered as a single patch and their individual areas were combined. Under these rigorous constraints, the number of patches, both in normal and in TTX-treated groups, dropped drastically, particularly in the arbors reconstructed from the youngest normal animals. For example, at P19, only two standard patches were found in two separate arbors (NN16 and NN17), and at P23 a single patch was found in one arbor (NN8). Figure $20 B$ illustrates the distribution of the areas of standard patches in normal and TTX-treated animals at the different ages. Due to the small sample of patches in normal animals at P19 and P23, statistical comparisons for the two experimental groups were made only for P29-P31 and P39 age groups. No statistically significant differences were found within or between groups, but the TTX arbors were more variable. Figure 21 illustrates examples of standard patches in arbors of normal and TTX-treated animals.

To further characterize patches and the degree of afferent segregation, we determined the density of terminals in the "interpatch space," that is, the zone between the standard patches. If segregation into eye-specific domains had occurred, then one would anticipate a low density within the interpatch space, as this zone should be occupied by afferents from the other eye. Alternatively, if segregation was incomplete, the density of terminals within the interpatch space should not differ from the minimum density of the patch. In normal animals, the average distance between patches was calculated to be $297.4 \mu \mathrm{m}$, slightly less than the separation of ocular dominance patches labeled autoradiographically or with WGA-HRP (Anderson et al., 1988). Thus, when single patches were analyzed, the density of the interpatch space was calculated at $150 \mu \mathrm{m}$ from the patch border (i.e., approximately $297.4 \mu \mathrm{m} \div 2$ ). For pairs of patches separated by more than $300 \mu \mathrm{m}$, the density of the interpatch space was calculated at $150 \mu \mathrm{m}$ from the patch border. However, for pairs of patches separated by less than $300 \mu \mathrm{m}$, the density of the interpatch space was calculated at the midpoint between the two patches. The density at the interpatch space was expressed as percentage difference from the minimum density of the stan- 
dard patch $\left(38 \mu \mathrm{m} / 1000 \mu \mathrm{m}^{2}\right)$. Figure $20 C$ illustrates the distribution of such reduction, in percentage terms, for the two experimental groups as a function of age. Note, in the group of normal animals, that at the youngest age (P19) the density in the interpatch space underwent a relatively small drop $(31.6 \%$ and $2.6 \%$ ) compared to older ages, though the number of patches at P19 and P23 is too small to allow a definite conclusion. At $\mathrm{P} 30 / 31$ the mean of the percentage reduction was $51.1 \%$, and at P39, 59.8\%. The lowest value in these normal animals corresponds to an interpatch density in arbor NN11 (Fig. 6) that failed to reveal, on the basis of a qualitative inspection, a clear segregation of the terminal arborization into distinct patches. In the TTX-treated group, no age-dependent significant differences were found. The means of the percentage reductions were $38.9 \%$ at $\mathrm{P} 23,34.8 \%$ at $\mathrm{P} 29$, and $32.9 \%$ at $\mathrm{P} 39$. The single large reduction in density at $\mathrm{P} 39(81.6 \%)$ was found in arbor TTX8 (Fig. 15) and corresponds to the drop in density between the large patch within the main, high-density arborization and the small ventral patch. Arbor TTX13 (Fig. 15), which on qualitative inspection appeared to bear two distinct patches, demonstrated instead the smallest drop in density in the interpatch space (13.2\%), indicating that only a small degree of segregation of the terminal arborization had occurred. In a comparison between normal and TTX-treated animals at P29-P39, the normal group showed a significantly greater drop in density in the interpatch space, compared to the TTX-treated group $(p=0.01)$.

\section{Discussion}

The main purpose of the present study was to examine, in the cat, the morphological changes of single geniculocortical arbors both during normal development and in the absence of impulse activity in the afferent visual pathways. Our work has been inspired by previous studies on the segregation of geniculocortical afferents during development and the role of activity during this process (LeVay et al., 1978; Stryker and Harris, 1986). These two studies used the autoradiographic method to reveal the pattern of innervation of area 17 by the whole geniculocortical projection. They demonstrated that from the second week to approximately the sixth week postnatally, the geniculocortical projections undergo a progressive eye-specific segregation and form ocular dominance domains, and that segregation is prevented when retinal impulse activity is blocked by bilateral intraocular injections of TTX.

The present analysis of single geniculocortical arbors was performed at three different ages within a 3 week period in which, during normal development, cortical projections have initiated and largely completed the eye-specific segregation (LeVay et al., 1978). Several conclusions may be drawn from this study. First, during normal development, from the third to the sixth week of age, the topographic extent of the cortex over which geniculocortical axons arborize generally decreases. Second, during this same period, a fundamental remodeling of the arbor occurs, characterized by the formation of distinct clumps of terminals. Third, there is a general growth and elaboration of the terminal arborization of geniculocortical axons. Fourth, blockade of the retinal activity alters the development of geniculocortical afferents. In TTX-treated animals the majority of arbors demonstrate a uniform distribution of terminals throughout layer IV, at an age when, in normal development, the terminal arborization has segregated into distinct patches of terminals. Finally, the complexity of the arbors in the TTX-treated animals suggests that growth and elaboration of the collateral branches is a con- tinuous and active process - even in the ahsence of spontaneous or visually driven activity in the afferent pathways.

It is appropriate to consider some potential flaws in our methodology. Our analysis is based on serial reconstruction of geniculocortical axons anterogradely labeled after PHA-L injection into the LGN. Since PHA-L was visualized with immunohistochemical methods, it is conceivable that incomplete labeling of the terminal arborization may have occurred due to inadequate diffusion of the incubation media in tissue sections. To minimize this problem we used long incubation times along with high concentrations of Triton X-100 (see Materials and Methods). Indeed, we are confident we have obtained a faithful picture of the total axonal terminal arborization in the gray matter, since collateral branches were clearly visible through the entire depth of the section. However, in the white matter, incomplete labeling of the axonal trunk may have occurred due to the greater compactness of white versus gray matter. Axonal trunks reconstructed within the cortical layers could rarely be traced far into the white matter. For this reason our study is an analysis of the arbors as some geniculocortical axons may give rise to more than one arbor by branching of the axonal trunks deep in the white matter (Humphrey et al., 1985a,b).

It should also be noted that the only parameter that has been analyzed both in absolute terms and proportionally to the growth of the brain is the linear measurement of the VD extension, the conclusions from which were similar before and after normalization. Though we have analyzed the coverage area of the terminal arborization and its density in absolute terms (i.e., without normalization for the areal or volumetric expansion of area 17, which we did not measure), we are confident that, like the VD extension, such normalization would not change any of our conclusions.

Finally, comparisons among axons labeled at different stages of development are potentially misleading if the labeled axons are of different classes at the different ages. Two prominent classes of geniculocortical axons that innervate layer IV are $\mathrm{X}$ and $Y$. While our anatomical findings cannot provide proof of the functional class of each axon, the sublaminar distribution of the arbors within layer IV suggests strongly that most of the arbors were of the $\mathrm{Y}$-class, arborizing only within the superficial portion of the layer (among the NN axons reconstructed in normal animals: four of five at P19, six of six at P23, five of five at $\mathrm{P} 30-\mathrm{P} 31$, and three of three at $\mathrm{P} 39$ ). The remaining axons arborized in both superficial and deep portion of layer IV and are presumably of the $\mathrm{X}$-class. We found that measurements of the axonal diameters in our material were unreliable because they were too close to the resolution limit of the light microscope.

\section{Maturation of geniculocortical arbors during normal development}

Although the maturation processes in the development of geniculocortical afferents are certainly evolving in a continuous fashion, our observations suggest that, during the age period considered here, they can be considered as subdivided into two phases: an initial immature phase and a later remodeling phase.

During the initial phase, the terminal arborization of geniculocortical arbors is formed by long, poorly ramified branches which sparsely innervate a wide topographic extent of area 17 . Although our analysis starts at P19, there is evidence that as early as P7, axonal terminal arborizations in layer IV, labeled with the lipophilic tracer DiI, have substantially the same morphol- 
ogy (Ghosh and Shat7, 1992). Similar features have also been reported for developing geniculocortical axons in primates (Florence and Casagrande, 1990) and in hamsters (Naegele et al., 1988).

One arbor reconstructed at P19 (Fig. 4) showed an unusually rich and elaborated terminal arborization that appears similar to a Golgi-impregnated arbor in a 2-week-old kitten described by LeVay and Stryker (1979). At present, there is not enough evidence available to indicate whether this kind of arbor forms a distinct class among the geniculocortical afferents or whether it indicates an extreme in the variability present at any age. It is possible that the remodeling of such axons during later stages of development entails predominantly segregative rather than growth processes, with the typical redistribution of collaterals in the form of distinct patches but no further great enrichment of the terminal arborization.

In the second remodeling phase, geniculocortical arbors demonstrate an increase of the arbor density with a concomitant remodeling of the terminal arborization and the formation of distinct patches of terminals. The most striking morphological changes, indicative of the progression of the second developmental phase, are found between P23 and P30/31. At the beginning of the fifth week of age (P30/31), the terminal arborizations show an increase in the total length and in the number of branch points, without a significant increase in coverage area. These factors lead to highly dense terminal arborizations characterized by short and spatially confined collaterals. Although we cannot provide data regarding the spatial distribution of the boutons and synapses within the arbor, the general anatomical characteristics of the terminal arborization at P30/31 appear well suited to concentrate activity onto a spatially restricted pool of postsynaptic cortical cells. It is noteworthy that cortical synaptogenesis is reported to increase drastically between the fourth and fifth week of age, is at its highest level by the fifth week, and then decreases reaching adult levels by 3 months (Cragg, 1975). Thus, during the second phase, between P23 and P30/ 31 , the growing density of collaterals in a single geniculocortical arbor and the exceedingly high number of synapses would appear to provide an ideal basis for the selective stabilization of geniculocortical synapses. Indeed, the convergence of densely clustered collaterals, carrying correlated visual information, onto the same set of postsynaptic neurons might provide the optimal situation for the reinforcement of eye-specific projections (Constantine-Paton et al., 1990; Singer, 1990).

The transformation of axons responsible for eye-specific segregation takes place during this second developmental phase between P23 and P30/31. At this age and subsequently, the patches appear well defined and form discrete areas of densely packed collaterals separated by terminal-free gaps, or are joined through a single, unramified connecting branch. A similar, welldefined clustering has been observed in geniculocortical afferents to area 17 in the adult cat (Freund et al., 1985; Humphrey et al., 1985a,b). Further evidence indicating that the remodeling of the terminal arborization in the form of patches occurs predominantly during the second developmental phase is provided by our quantitative analysis of the density profiles of the terminal arborization. Such analysis has confirmed that high-density, adult-like patches of collaterals, defined as "standard patches" in Results, are found in all axons after P30/31, but only sporadically at P19 and P23. In addition, the analysis of the change in arbor density between a patch and its surrounding regions has revealed that this gradient is steep at P30/31 and
$\mathrm{P} 39$, and relatively shallow at $\mathrm{P} 19$. The small difference in density between patches and the regions between them indicates that the few patches present at younger ages are not equivalent to the clusters of terminals with clearly defined borders typical of older ages. Rather, the shallow gradient is indicative that the final sculpting of the patch, involving the selective elimination of branches in regions destined to be innervated by the afferents serving the other eye is not yet fully accomplished. Unlike arbors at P30/31 and P39, the majority of the arbors at P19 and P23 are rather homogeneously distributed in layer IV.

It should be noted, however, that although the segregation process is accelerated during the remodeling phase and appears almost complete by $\mathrm{P} 30 / 31$, its onset and progression have certainly been initiated at younger ages. Quantitative analysis of the arbor density profiles has shown that even at the youngest age (P19) the terminal arborizations are characterized by small nonuniformities in density. However, the density values of the previously defined "high-density zones" within each arbor are significantly lower than the density levels found at P30/31 and P39. At P19 and P23, these high-density zones might represent either stochastic fluctuations in density of no significance, or, more plausibly, immature forms of the adult ocular dominance domains. In the latter case, these immature "patches" of terminals may acquire in the following 7-10 d a higher density and better defined borders by increasing the density gradient between the patch and the surrounding zones.

LeVay et al. (1978) have demonstrated with autoradiography that the geniculocortical projection in the cat segregates into distinct patches in a progressive fashion from an initially diffuse state. At P15 and at younger ages, the autoradiographic labeling in layer IV is continuous and uniform. By the beginning of the fourth week (P22) a periodic fluctuation of labeling is evident that becomes more distinct at P33 and by P39 is nearly as clear as in the adult (Shatz et al., 1977; LeVay et al., 1978). If we consider the presence of adult-like patches as the sole expression of the segregation process, then our observations appear to be in agreement with the autoradiographic analysis. In fact, few adult-like patches are seen at P19 and P23, at the time when only a small periodic fluctuation in the density of the autoradiographic labeling was found (for technical reasons only in the projection serving the ipsilateral eye).

Geniculocortical arbors reconstructed at P39 present substantially the same morphological characteristics found at P30/ 31 , that is, high density of collateral branches and many welldefined, adult-like patches. However, by this age the area of layer IV covered by the terminal arborization has expanded significantly.

This expansion of coverage area is accompanied by a decrease rather than an increase in the ventrodorsal extent of the arbor, which corresponds to a reduction in the topographic extent of cortex that the arbor may contact. It is worth noting that some other measures of arbor "area" are more similar to our measure of "VD extention" rather than our measure of coverage area (Florence and Casagrande, 1990). Their measure of "ared" includes large regions in which there are gaps in the arbor, regions in which cortical cells would not be contacted by the arbor. Their "area" measure is tremendously influenced by the presence or absence of a single unramified branch extending in a direction away from the main arbor. Our measure of coverage area, like the area measurements of Humphrey et al. (1985a,b), includes only the area of cortex within which cells could be contacted by the arbor. 
While the arbors have attained an adult-like form by P39, they appear likely to continue to expand after that time. The average coverage area in area 17 of the adult cat $\left(0.7 \mathrm{~mm}^{2}\right.$ for $\mathrm{X}$ - and $1.3 \mathrm{~mm}^{2}$ for Y-type afferents; Humphrey et al., 1985a) is considerably greater than that of our arbors reconstructed at P39. Even considering the possibility that we have reconstructed arbors and not whole axons, the difference between the two ages suggests strongly that an expansion and elaboration of the arbor takes place after P39. Consistent with this interpretation is evidence that in area 18, the surface area of $Y$-axons increases between the fourth and fifth weeks and adulthood (Friedlander and Martin, 1989). Part of the increase in coverage area may be due to the overall growth of area 17, but much seems likely to be due to a genuine elaboration of the terminal arborization.

\section{Influence of retinal activity on the development of geniculocortical arbors}

In the TTX-treated group, bilateral retinal blockade was started at P13 or at P17 (see Table 1). At both ages, the geniculocortical projection is homogeneously distributed throughout layer IV and does not demonstrate any fluctuation in the autoradiographic label density, suggesting that the segregation in eye-specific domains is not yet manifest (LeVay et al., 1978). Based on our results and those of Ghosh and Shatz (1992), we can envisage that at these ages geniculocortical axons are still characterized by low-density, poorly ramified terminal arborizations. From this initial scenario, how does the geniculocortical arbor morphology evolve in the absence of retinal impulse activity? In the experiments that inspired the present study, Stryker and Harris (1986) observed that when the TTX treatment was initiated at ages similar to those chosen in our experiments, the geniculocortical afferents failed to segregate into eye-specific domains. Using both autoradiographic methods and electrophysiological recordings, these authors have demonstrated that after retinal blockade, the geniculocortical projection extended homogeneously through layer IV and almost all cortical neurons were driven by both eyes. Three alternate hypotheses on anatomical substrates of these results may be advanced. Specifically, the absence of activity in the afferent pathway may arrest the general growth and development of the geniculocortical projections. Under this hypothesis, the arbors would maintain the immature features present at the time TTX treatment was initiated. A second hypothesis posits that retinal activity blockade induces profuse nonspecific growth, but does not affect the cellular recognition based on eye of origin. Under this hypothesis, eye-specific segregation fails to take place only because afferents are so strongly stimulated to grow that they become too large to be confined to an ocular dominance column. Alternatively, the absence of activity may interfere predominantly with recognition mechanisms. That is, the geniculocortical afferents may continue to grow and elaborate their terminal arborization, but fail to aggregate in eye-specific clusters. Our results are consistent with the last hypothesis.

In the following paragraphs we review first the evidence that geniculocortical arbors in the absence of activity are not "frozcn" in a juvcnilc statc, but continuc to grow and elaborate collateral branches. Next, we consider the fluctuation in density within the terminal arborizations and discuss our observations relative to previously reported autoradiographic findings. Finally, we will consider our observations in terms of possible mechanisms underlying the process of afferent segregation in area 17 .
Growth of geniculocortical arbors in the absence of retinal activity. The detailed analysis of various quantitative parameters from the reconstructed arbors suggests that the growth of geniculocortical projections in TTX-treated animals proceeds in a manner overall similar to normal developing animals. Specifically, the density of the terminal arborization and the number of branch points, indicating the complexity of the arborization, have values similar to those found in normal animals. In addition, the analysis of two other parameters, total length and surface area of the arbor, appears to indicate that the growth was moderately enhanced. The total length of the terminal arborizations, taken as an index of growth, although widely variable at all ages, was on average somewhat greater in the TTXtreated compared to the normal groups. Further, the highest values of the arbor total length were found, at each age, among the TTX-treated animals. On average, the surface areas of arbors were also larger in TTX-treated animals than in normals, thus maximizing the opportunity for binocular mixing between the afferents serving the two eyes. This finding is in agreement with physiological findings of Stryker and Harris (1986).

Results from the P23 TTX-treated animal indicate that effects of the absence of activity are manifest within a few days from the beginning of TTX treatment. In this animal, the total length, density, and degree of ramification of the terminal arborization were all significantly larger than in the P23 age-matched control, even though this animal had only $6 \mathrm{~d}$ of TTX treatment. The effects of TTX were independent of the duration of the treatment, and no age-dependent developmental modifications in the structure of geniculocortical arbors were found, suggesting that the prompt effects of TTX are then maintained as long as the treatment is continued.

There is some controversy in the literature on the effects of activity blockade on the general growth of neural pathways (reviewed in Frank, 1987; Schmidt and Tieman, 1989). The hypothesis that TTX promotes growth comes from in vitro studies demonstrating that activity arrests neurite outgrowth in cultured neurons of both Heliosoma (Cohan and Kater, 1986) and mouse dorsal root ganglion (Fields et al., 1990), and from in vivo experiments demonstrating that TTX blockade promotes profuse sprouting in rat and frog neuromuscular junction (Brown and Ironton, 1977; Diaz and Pecot-Dechavassine, 1989). TTX-induced activity blockade appears also to promote sprouting and growth in the tectum of the three-eyed frog, and causes both degradation of the patches and sprouting of retinotectal fibers (Reh and Constantine-Paton, 1985). In contrast, other studies suggest that TTX treatment retards neuronal differentiation (Riccio and Matthews, 1985a,b; Kalil et al., 1986; Hsiao et al., 1987). An alternative interpretation of the disputed growthpromoting effects observed in TTX experiments is that activity per se is instrumental in error correction, such as eliminating misdirected branches and misplaced synapses. In the absence of activity, nonselective connections would be maintained, the refinement of the pattern of connections would not occur, and neural projections remain disordered and disorganized. Examples in support of this interpretation are found in several systems during both normal development and regencration (O'Leary et al., 1986; Shatz and Stryker, 1988; Thompson and Holt, 1989; Schmidt and Buffard, 1990). Our observations in TTX-treated animals appear to confirm a moderate growthpromoting effect of the lack of impulse activity. However, it is noteworthy that a distinctive feature of arbors reconstructed from all TTX-treated animals was the considerable variability 
of all parameters analyzed, at all ages studied, consistent with the hypothesis of the importance of impulse activity in organizing and coordinating the growth of the terminal arborizations. Indeed, during normal development, activity may modulate, in specific fashion, the function of transmitters and neuromodulators (Hendry and Jones, 1988), and may also differentially affect various types of geniculocortical afferents, depending on the frequency and pattern of spontancous activity (Fields et al., 1990).

Density profiles of geniculocortical arbors in the absence of retinal activity. While geniculocortical arbors in normal and TTX-treated animals have comparable density values, the analysis of the density gradient between the standard patch and the surrounding regions in TTX-treated animals demonstrates that the majority of these patches, though highly dense, appear as minor fluctuations on the marginally less dense background formed by the rest of the arborization. Thus, distinct, wellsculpted patches are not a common feature of geniculocortical arbors developed in the absence of impulse activity. The absence of clustering observed in TTX-treated animals (Stryker and Harris, 1986) may be due either to insensitivity to shallow fluctuations in label density in transneuronal autoradiographic labeling experiments, or to a random distribution of geniculocortical arbors in layer IV such that density fluctuations cancel cach other, rendering the autoradiographic labeling uniform.

The question remains as to why a small number of welldefined patches are found in TTX-treated animals. We do not have an easy answer in this regard. The observation that rules out the possibility that the segregation might have occurred in the P39 group when TTX treatment was interrupted to allow recordings from, and PHA-L injections into, the LGN (see Materials and Methods) is that similar apparent patches are present at P23 and P29 as well as at P39. We suggest that these patches may result from random growth, which sometimes forms clusters by chance. Alternatively, one may consider that afferent segregation can, in small part, use other cues than those conveyed by activity.

\section{The process of segregation of geniculocortical afferents}

The current hypothesis for the process of segregation of the geniculocortical projection into alternate eye-specific domains proposes that the terminal arborizations of the individual axons serving each eye are initially overextended, homogeneous, and largely overlapping those of the other eye. Subsequently, remodeling into alternate distinct clusters occurs by the elimination of mispositioned collaterals from cortical sites innervated predominantly by the other eye along with a growth and elaboration of synapses in appropriate position (LeVay et al., 1978; LeVay and Stryker, 1979). Our observations largely support this hypothesis, but put increased emphasis on the selective growth of arbors in the territory within ocular dominance patches. Overall, arbors in young animals were widely extended but sparse. The average extension of arbors along the ventrodorsal axis of area 17 decreases from the ages of P19/23 to P30/39. Between $\mathrm{P} 23$ and P30/31 the average branch length is also dramatically reduccd, whilc there is an incrcasc of the total length of the axonal membrane. This suggests that long, far reaching branches either have developed new strategically positioned short collaterals, so as to give rise to the typical clusters, or they have been selectively eliminated and new collaterals have been added to other portions of the terminal arborization. A possible example of remodeling by elimination of collaterals could be represented by arbor $\mathrm{L}$ (Figs. 8,9 ) reconstructed in a normal animal at P39. The terminal arborization ramifies within two eye-specific patches, while the axonal trunk enters into layer IV between the two patches, in a region presumably innervated by the other eye. We can envisage that during an earlier phase of development, a few branches were located in this region, above the axonal trunk, and they were later eliminated.

Normal devclopment thus appears to involve both selective elimination of widely extended branches and considerable growth and elaboration.

\section{References}

Anderson PA, Olivarria J, Van Sluyters RC (1988) The overall pattern of ocular dominance bands in cat visual cortex. J Neurosci 8:21832200.

Arnett DW (1978) Statistical dependence between neighboring retinal ganglion cells in the goldfish. Exp Brain Res 32:49-53.

Arnett DW, Spaker TE (1981) Cross-correlation analysis of the maintained discharge of rabbit retinal ganglion cells. J Physiol (Lond) 317: 29.

Brown MC, Ironton R (1977) Motor neurone sprouting induced by prolonged tetrodotoxin block of nerve action potentials. Nature 265: $459-461$.

Changeux J, Danchin A (1976) Selective stabilization of developing synapses as a mechanism for the specification of neuronal networks. Nature 264:705-711.

Cohan CS, Kater SB (1986) Suppression of neurite elongation and growth cone motility by electrical activity. Science $232: 1638-1640$.

Constantine-Paton M, Cline HT, Debski E (1990) Patterned activity, synaptic convergence, and the NMDA receptor in developing visual pathways. Annu Rev Neurosci 13:129-154.

Cragg BG (1975) The development of synapses in the visual system of the cat. J Comp Neurol 160:147-166.

Diaz J, Pecot-Dechavassine M (1989) Terminal nerve sprouting at the frog neuromuscular junction induced by prolonged tetrodotoxin blockade of nerve conduction. J Neurocytol 18:39-46.

Edwards DL, Grafstein B (1984) Intraocular injection of tetrodotoxin in goldfish decreases fast axonal transport of $\left[\mathrm{H}^{3}\right]$ glucosamine-labeled materials in optic axons. Brain Res 299:190-194.

Ferster D, LeVay SM (1978) The axonal arborizations of lateral geniculate neurons in the striate cortex of the cat. J Comp Neurol 182: 923-944

Fields RD, Neale EA, Nelson PG (1990) Effects of patterned electrical activity on neurite outgrowth from mouse sensory neurons. J Neurosci 10:2950-2964.

Florence SL, Casagrande VA (1990) Development of geniculocortical axon arbors in a primate. Vis Neurosci 5:291-309.

Frank E (1987) The influence of neuronal activity on patterns of synaptic connections. Trends Neurosci 10:188-190.

Freund TF, Martin KAC, Whitteridge D (1985) Innervation of cat visual area 17 and 18 by physiologically identified X-and Y-type thalamic afferents. I. Arborization patterns and quantitative distribution of postsynaptic elements. J Comp Neurol 242:263-274.

Friedlander MJ, Martin KA (1989) Development of Y-axon innervation of cortical area 18 in the cat. J Physiol (Lond) 416:183-213.

Friedlander MJ, Lin C-S, Stanford LR, Sherman SM (1981) Morphology of functionally identified neurons in lateral geniculate nucleus of the cat. J Neurophysiol 46:80-129.

Gerfen CR, Sawchenko PE (1984) An anterograde ncuroanatomical tracing method that shows the detailed morphology of neurons, their axons and terminals: immunohistochemical localization of an axonally transported plant lectin, Phaseolus vulgaris leucoagglutinin (PHA-L). Brain Res 290:219-238.

Ghosh A, Shatz CJ (1992) Pathfinding and target selection by developing geniculocortical axons. J Neurosci 12:39-55.

Hebb DO (1949) Organization of behaviour. New York: Wiley.

Hendry SHC, Jones EG (1988) Activity-dependent regulation of GABA expression in the visual cortex of adult monkey. Neuron 1:701-712.

Hsiao CF, Dubin MW, Kalil RE (1987) Rearing cats with TTX-blockade of retinal ganglion cells (RGC) activity affects the morphological development of LGN neurons. Invest Ophthal Vis Sci [Suppl] 28:1.

Hubel DH, Wiesel TN (1970) The period of susceptibility of the 
physiological effects of unilateral eye closure in kittens. J Physiol (Lond) 206:419-436.

Hubel DH, Wiesel TN (1972) Laminar and columnar distribution of geniculocortical fibers in the macaque monkey. J Comp Neurol 146: $421-450$.

Hubel DH, Wiesel TN (1977) Functional architecture of the macaque monkey visual cortex. Proc R Soc Lond [Biol] 198:1-59.

Humphrey AL, Sur M, Ulrich DJ, Sherman SM (1985a) Projection patterns of individual $\mathrm{X}$ - and $\mathrm{Y}$-cell axons from the lateral geniculate nucleus to cortical area 17 in the cat. J Comp Neurol 233:159-189.

Humphrey AL, Sur M, Uhlrich DJ, Sherman SM (1985b) Termination patterns of X-and Y-cell axons in the visual cortex of the cat: projections to area 18 , to the $17 / 18$ border region, and to both areas 17 and 18. J Comp Neurol 233:190-212.

Jones EG (1990) The role of afferent activity in the maintenance of primate neocortical function. J Exp Biol 153:155-176.

Kalil RE (1990) The influence of action potentials on the development of the central visual pathway in mammals. J Exp Biol 153:261-276.

Kalil RE, Dubin MW, Scott GL, Stark La (1986) Elimination of action potentials blocks the structural development of retinogeniculate synapses. Nature 323:156-158.

LeVay SM, Gilbert CD (1976) Laminar patterns of geniculocortical projections in the cat. Brain Res 113:1-19.

LeVay SM, Stryker MP (1979) The development of ocular dominance columns in the cat. In: Society for Neuroscience Symposium: Aspects of developmental neurobiology (Ferrendelli JA, ed), pp 83-98. Bethesda, MD: Society for Neuroscience.

LeVay SM, Stryker MP, Shatz CJ (1978) Ocular dominance columns and their development in layer IV of the cat's visual cortex. A quantitative study. J Comp Neurol 179:559-576.

LeVay SM, Wiesel TN, Hubel DH (1980) The development of ocular dominance columns in normal and visually deprived monkeys. J Comp Neurol 191:1-51.

Lund JS, Henry GH, Macqueen CL, Harvey AR (1979) Anatomical organization of the primary visual cortex (area 17) of the cat. A comparison with area 17 of the macaque monkey. J Comp Neurol 184:599-618.

Mastronarde DN (1983a) Correlated firing of cat retinal ganglion cells. I. Spontaneously active inputs to $X$ - and Y-cells. J Neurophysiol 49: 303-324.

Mastronarde DN (1983b) Correlated firing of cat retinal ganglion cells. II. Rcsponsc of X- and Y-cells to single quantal response. J Neurophysiol 49:325-345.

Miller KD, Stryker MP (1990) The development of ocular dominance columns: mechanisms and models. In: Connectionist modeling and brain function: the developing interface (Hanson SJ, Olson CR, eds), pp 255-350. Cambridge, MA: MIT Press.

Naegele JR, Jhaveri S, Schneider GE (1988) Sharpening of topographical projections and maturation of geniculocortical axon arbors in the hamster. J Comp Neurol 277:593-607.

O'Leary DDM, Crespo D, Fawcett J, Cowan WM (1986) The effects of intraocular tetrodotoxin on the postnatal reduction in the number of optic nerve axons in the rat. Dev Brain Res 30:96-103.

Otsuka R, Hassler R (1962) Uber Aufbau und Gliederung der corticalen Sehesphäre bei der Katze. Arch Psychiatr Zeitsch Neurol 203: 212-234.

Passera A, Fulks S, Schneider GE, Ayres S, Jhaveri S, Erzurumlu RS (1988) The M.I.T. "Neurotrace" system for microcomputer-aided microscopy. Soc Neurosci Abstr 14:550.

Purves D, Lichtman JW (1984) Principles of neuronal development. Sunderland, MA: Sinauer.

Rakic P (1976) Prenatal genesis of connections subserving ocular dominance in the rhesus monkey. Nature 261:467-471.

Reh TA, Constantine-Paton M (1985) Eye-specific segregation requires neural activity in three-eyed Rana pipiens. J Neurosci 5:11321143.

Riccio RV, Matthews MA (1985a) Effects of intraocular tetrodotoxin on the postnatal devclopment of the dorsal lateral geniculate nucleus of the rat: a Golgi analysis. J Neurosci Res 17:440-451.

Riccio RV, Matthews MA (1985b) Effects of intraocular tetrodotoxin on dendritic spines in the developing rat visual cortex: a Golgi analysis. Dev Brain Res 19:173-182.

Riccio RV, Matthews MA (1985c) The effects of intraocular injection of tetrodotoxin on fast axonal transport of $\left[\mathrm{H}^{3}\right]$ proline- and $\left[\mathrm{H}^{3}\right]$ fucoselabeled materials in the developing rat optic nerve. Neuroscience 16 : 1027-1039.

Schmidt J, Buffard M (1990) Activity-driven sharpening of the regenerating retinotectal projections: effects of blocking or desynchronizing activity on the morphology of individual regenerating arbors. J Neurobiol 21:900-917.

Schmidt J, Tieman SB (1989) Activity, growth cones and the selectivity of visual connections. Comm Dev Neurobiol 1:11-28.

Shatz CJ, Luskin MB (1986) Relationship between the geniculocortical afferents and their cortical target cells during development of the cat. J Neurosci 6:3655-3668.

Shatz C, Stryker MP (1988) Prenatal tetrodotoxin infusion blocks segregation of retinogeniculate afferents. Science 242:87-89.

Shatz C, Lindstrom SH, Wiesel TN (1977) The distribution of afferents representing the right and left eyes in the cat's visual cortex. Brain Res 131:103-116.

Singer W (1990) The formation of cooperative cell assemblies in the visual cortex. J Exp Biol 153:177-197.

Stent GS (1973) A physiological mechanism for Hebb's postulate of learning. Proc Natl Acad Sci USA 70:997-1001.

Stryker MP, Harris WA (1986) Binocular impulse blockade prevents the formation of ocular dominance columns in cat visual cortex. $J$ Neurosci 6:2117-2133.

Thompson I, Holt C (1989) Effects of intraocular tetrodotoxin on the development of the retinocollicular pathway in the Syrian hamster. J Comp Neurol 282:371-388.

Tusa RJ, Palmer LA, Rosenquist AC (1978) Retinotopic organization of area 17 (striate cortex) of the cat. J Comp Neurol 177:213-236. 